\title{
Computation of General Inner-Outer and Spectral Factorizations
}

\author{
Cristian Oară, and Andras Varga, Senior Member, IEEE
}

\begin{abstract}
In this paper we solve two problems in linear systems theory: the computation of the inner-outer and spectral factorizations of a continuous-time system considered in the most general setting. We show that these factorization problems rely essentially on solving for the stabilizing solution a standard algebraic Riccati equation of order usually much smaller than the McMillan degree of the transfer function matrix of the system. The proposed procedures are completely general being applicable for a polyno$\mathrm{mial} / \mathrm{proper} / \mathrm{improper}$ system whose transfer function matrix could be rank deficient and could have poles/zeros on the imaginary axis or at infinity. As an application we discuss the extension to rational matrices of the complete orthogonal decomposition of a constant matrix. Numerical refinements are discussed in detail. To illustrate the proposed approach several numerical examples are also given.
\end{abstract}

Keywords - Inner-outer factorization; spectral factorization; rational matrices; descriptor systems; system zeros; singular matrix pencils; numerical algorithms

\section{INTRODUCTION}

In this paper we address two related problems in linear system theory: the computation of the inner-outer factorization and of the spectral factorization of a rational matrix. A large number of quite different types of factorizations are covered in the literature under these names but most of them impose additional restrictive assumptions that rule out the difficult cases. The two general factorization problems that we solve in this paper are now stated.

\section{A. Problem statement}

Throughout the paper we consider matrices with real coefficients as this is the typical case in control applications. Moreover, from a numerical viewpoint the real case is slightly more difficult than the complex case, and the latter follows by minor modifications of notation and definitions from the former. By $\mathbb{C}, \mathbb{C}^{-}, \mathbb{C}^{+}$, and $\mathbb{C}_{0}$, we denote the complex plane, the open left half-plane, the open right half-plane, and the imaginary axis, respectively, and let $\overline{\mathbb{C}}:=\mathbb{C} \cup\{\infty\}, \overline{\mathbb{C}^{-}}:=\mathbb{C}^{-} \cup \mathbb{C}_{0} \cup\{\infty\}$, $\overline{\mathbb{C}^{+}}:=\mathbb{C}^{+} \cup \mathbb{C}_{0} \cup\{\infty\}$. Here "overbar" denotes closure. We call a rational matrix $G(s)$ injective if it has full column rank for almost all $s$ and surjective if it has full row rank for almost all $s$. For a rational matrix with real coefficients $G(s)$ we define its conjugate $G^{*}(s):=G^{T}(-s)$. We call a

C. Oară is with the Faculty of Automatic Control and Computers, University Polytechnica Bucharest, 34 Austrului, RO 73115 , Bucharest, Romania (e-mail: oara@mediasat.ro), Tel/Fax: +40 1 3234 234. His work has been made possible by a Fellowship of the Alexander von Humboldt Foundation, Bonn.

A. Varga is with the Institute of Robotics and Mechatronics, German Aerospace Center, DLR-Oberpfaffenhofen, D-82234, Wessling, Germany (e-mail: Andras.Varga@dlr.de). real rational matrix $M(s)$ all-pass if it is square and satisfies $M^{*}(s) M(s)=I$, and we call it inner if it is analytic in $\overline{\mathbb{C}}^{+}$and satisfies $M^{*}(s) M(s)=I$. Note that an inner matrix need not to be square, yet it must be injective.

Inner-Outer Factorization Problem. Given $G(s)$ an arbitrary rational matrix analytic in $\overline{\mathbb{C}}_{+}$(i.e., proper and stable), determine two rational matrices $G_{i}(s)$ and $G_{o}(s)$ such that

$$
G(s)=G_{i}(s) G_{o}(s),
$$

where $G_{i}(s)$ is inner, $G_{o}(s)$ is analytic in $\overline{\mathbb{C}}^{+}$and surjective, and has a right inverse analytic in $\mathbb{C}^{+} . G_{o}(s)$ is called an outer factor, and (1) defines an inner-outer factorization of $G(s)$. By a slight abuse of terminology, we shall consider in this paper also inner-outer factorizations for systems which are analytic in $\mathbb{C}^{+}$(also called "weak-stable", allowing for $G(s)$ to be improper and have poles on $\left.\mathbb{C}_{0}\right)$. In this case we require the inner factor to be analytic in $\overline{\mathbb{C}}^{+}$, but we impose correspondingly analyticity of $G_{o}(s)$ only in $\mathbb{C}^{+}$ and for the "outer" factor to have a right inverse analytic in $\mathbb{C}^{+}$.

Spectral Factorization Problem. Given $G(s)$ an arbitrary real rational matrix, determine a real rational matrix $G_{o}(s)$ such that

$$
G^{*}(s) G(s)=G_{o}^{*}(s) G_{o}(s),
$$

where $G_{o}(s)$ is surjective and analytic in $\mathbb{C}^{+}$and has a right inverse analytic in $\mathbb{C}^{+} . G_{o}(s)$ is called a spectral factor of $G(s)$ and (2) defines a spectral factorization of $G(s)$.

We should mention at this point that the spectral factorization problem as formulated in [48] or [1], is more general. There, a real rational matrix $\Phi(s)$ is given - also called spectral density - satisfying $\Phi(s)=\Phi^{T}(-s) \geq 0, \forall s \in \overline{\mathbb{C}}_{0}$, and one seeks a real rational matrix $G_{o}(s)$ such that

$$
\Phi(s)=G_{o}^{*}(s) G_{o}(s),
$$

where the spectral factor $G_{o}(s)$ must satisfy the same requirements as in our formulation. The only difference between the above mentioned problem and the one that we consider is that in our formulation $\Phi(s)$ is in a prefactorized form

$$
\Phi(s)=G^{*}(s) G(s)
$$

which is the typical case in control related applications (see [50]). The factor $G(s)$ in (3) is an arbitrary rational matrix.

The inner-outer and the spectral factorization problems as formulated above are strongly connected in the following sense. Assume temporarily $G(s)$ is analytic in $\mathbb{C}^{+}$and let (2) be a spectral factorization of it. Then one can prove that $G_{i}(s):=G(s) G_{o}^{\#}(s)$ is inner, where $G_{o}^{\#}(s)$ is a right 
inverse of $G_{o}(s)$ analytic in $\mathbb{C}^{+}$, and $G_{i}(s)$ together with $G_{o}(s)$ define an inner-outer factorization (1) of $G(s)$. Conversely, if (1) is an inner-outer factorization of $G(s)$ then we get immediately that $G_{o}(s)$ is a spectral factor of $G(s)$ satisfying (2). Notice that the spectral factorization problem can be posed for a more general class of systems than the inner-outer factorization problem including systems $G(s)$ with arbitrary poles in $\overline{\mathbb{C}}$.

Inner-outer and spectral factorizations appear throughout control systems, identification, signal processing, and circuit theory, and it is surely hopeless to give here a short but still comprehensive account on all applications in which they occur. Instead, we shall give a historical perspective on the computational methods for solving these two related problems at various levels of generality.

\section{B. A historical perspective}

The spectral factorization problem appeared first in Wiener filtering and in solving integral equations with rational symbols (see [4] for a comprehensive account of these applications and for relevant references). These methods became popular in the engineering literature starting with the celebrated paper of Youla [48] in which the solvability of the most general spectral factorization problem is proved, and with [33] which showed the connection between linear quadratic control and spectral factorization problems. The first paper to derive formulas for the spectral factors by using algebraic manipulations is [1] where the generality is restricted only by excluding the presence of poles and zeros of $\Phi(s)$ on the imaginary axis. Unfortunately, these manipulations are based on highly unreliable algorithms including operations on polynomial matrices and Smith form. Difficulties in solving numerically the spectral factorization problem are related to the occurrence of poles and zeros on the imaginary axis and at infinity, and to the non-invertibility of $\Phi(s)$ (for a detailed discussion see [11]). If none of these "pathological" elements are present, the computation of the spectral factor reduces to solving a standard continuous-time algebraic Riccati equation for which numerically reliable algorithms were proposed by Laub [25]. During the last three decades, tens of papers addressed the computation of the spectral factorization and strove to include some of the "difficult" cases. For example, in [24] "weak-stabilizing" solutions to Riccati equations are introduced to deal with imaginary axis zeros, but no algorithm is given. The case in which $\Phi(s)$ in (3) has not full rank but has no zeros on the imaginary axis can be tackled by various types of algebraic Riccati systems as those introduced by Ionescu and Oară in [20], [19], [21] and for which numerically-sound algorithms based on Kronecker-like forms for singular pencils [37], [5], [29] have been also proposed. When $\Phi(s)$ has zeros at infinity, the Riccati equation can not be even written down and has to be replaced with matrix inequalities as in [47]. Perhaps the most general approach to the spectral factorization problem is of Clements [11], [12], where the case in which $\Phi(s)$ may have simultaneously arbitrary rank and imaginary axis zeros and poles is considered (still
$\Phi(s)$ must be proper, i.e. must have no poles at infinity). Nevertheless, the methods derived there, although some of them algorithmic and very general, are far from numerically-sound computational procedures. Endeavors to include the improper case are made in [13] and [22]. The complete numerical solution for the case of an arbitrary $\Phi(s)$ which is not in a prefactorized form implies a symmetric separation of the complete Kronecker structure of an arbitrary Hermitic or Hamiltonian pencil. This comes down to the derivation of a symmetric version of the general staircase algorithm of Van Dooren [37] and no solution to this algorithmic problem has emerged so far despite the many attempts (see [26] for a comprehensive account on structure-preserving algorithms).

As for computing inner-outer factorizations, difficulties associated with the same "pathological" elements are encountered, and two classes of methods have been proposed so far. The first class of methods essentially solves the associated spectral factorization problem and thus relies on computing stabilizing solutions of certain Riccati or generalized Riccati equations [14]. The most general methods in this class can deal with $G(s)$ of arbitrary rank but without zeros on the imaginary axis (including infinity). In this respect [21] treat the most general cases. If such zeros are present, semi-stabilizing (or weak stabilizing) solutions instead of stabilizing solutions to Riccati equations have to be computed and this leads to the same numerical difficulties as for the spectral factorization problem. Moreover, as will become clear from this paper, the redundancy caused by solving unnecessarily high order Riccati equations (of order equal to the McMillan degree of $G(s)$ ) represents the principal disadvantage of these methods from a numerical point of view. Even worse, the redundancy in all the above methods usually leads to non-minimal representations of the resulting inner factors.

The second class of methods avoids the solution of Riccati equations and is based on conjugation of unstable zeros to stable locations by multiplying $G(s)$ to the left with suitable square elementary all-pass factors. However, this class of methods provides the solution to the inner-outer factorization problem only in the very particular case in which $G(s)$ is surjective. The general technique of zero dislocation is described by Van Dooren [40]. All the methods in this class can perform recursively, by dislocating one real zero or a pair of complex conjugated zeros at a time with a factor of McMillan degree 1 or 2, respectively. The first algorithm to compute recursively the inner-outer factorization of a surjective proper rational matrix, either in continuous or discrete-time, is implicitly contained in the report [39] published later on as [40], where a more general class of factorization problems is discussed. Apparently unaware of [39], Kimura [23] solves the inner-outer factorization problem in the more particular case of a square invertible continuous-time system. His method implies explicit inversion of $G(s)$ and recursively dislocating the poles of $G^{-1}(s)$ by a method that he called "conjugation". Algorithms based on a similar idea, but without explicitly forming the inverse system, have been proposed by Zhang 
and Freudenberg [49]. A recursive algorithm which solves the inner-outer factorization problem for improper surjective $G(s)$ has been recently proposed in [41]. As explained above, the important limitation of all these conjugation techniques is that they can solve only the case in which $G(s)$ is surjective, yet they can tackle zeros on the imaginary axis including infinity.

Summarizing the above discussion, the methods in the first class can not deal with zeros on the imaginary axis while the methods in the second class can not solve problems with $G(s)$ non-surjective. No numerical methods are available for nonsurjective matrices featuring zeros on the imaginary axis and at infinity, although this situation occurs frequently in practice.

\section{Outline of the proposed approach}

Let $G(s)$ be an arbitrary $p \times m$ real rational matrix of rank $r$. Our approach to the inner-outer and spectral factorization problems rely on two basic factorizations.

\section{Row compression by all-pass factors}

We factorize an arbitrary $G(s)$ as

$$
G(s):=G_{a}(s) \widetilde{G}(s),
$$

where $G_{a}(s)$ is square inner and $\widetilde{G}(s)$ is row compressed, i.e., the trailing $p-r$ rows of $\widetilde{G}(s)$ are zero

$$
\widetilde{G}(s)=\left[\begin{array}{c}
\widetilde{G}_{1}(s) \\
O
\end{array}\right] \begin{aligned}
& \} r \\
& \} p-r
\end{aligned}
$$

This comes down to computing $G_{a}^{-1}(s)$ such that in the product $G_{a}^{-1}(s) G(s)$ all minimal indices to the left of $G(s)$ are canceled (are all made zero) while zeros in $\mathbb{C}^{-}$are introduced instead. We choose $G_{a}(s)$ to have the smallest possible McMillan degree $n_{\ell}$ which is equal to the sum of all left minimal indices of $G(s)$. The computation of $G_{a}(s)$ amounts to solving for the stabilizing solution a standard Riccati equation of order $n_{\ell}$. Combining (5) and (4) we get

$$
G(s)=G_{i 1}(s) \widetilde{G}_{1}(s)
$$

where $G_{a}(s)=\left[\begin{array}{ll}G_{i 1}(s) & G_{i 2}(s)\end{array}\right], G_{i 1}(s)$ is inner, $\widetilde{G}_{1}(s)$ is surjective and has the same zeros in $\overline{\mathbb{C}}^{+}$as $G(s)$, and its zeros in $\mathbb{C}^{-}$are the union of the zeros in $\mathbb{C}^{-}$of $\widetilde{G}(s)$ with the zeros of $G_{a}^{-1}(s)$.

\section{Dislocation of zeros by all-pass factors}

We factorize a surjective $G(s)$ as

$$
G(s)=G_{a}(s) \widetilde{G}(s)
$$

where $G_{a}(s)$ is square inner and $\widetilde{G}(s)$ is surjective and has no zeros in $\mathbb{C}^{+}$(i.e., $\widetilde{G}(s)$ is outer). This comes down to computing $G_{a}^{-1}(s)$ such that in the product $G_{a}^{-1}(s) G(s)$ all $\mathbb{C}^{+}$zeros of $G(s)$ are canceled and reflected into symmetric positions in $\mathbb{C}^{-}$(with respect to the imaginary axis). Again, we chose $G_{a}(s)$ to have the smallest possible McMillan degree which is equal to the number $n_{b}$ of zeros of $G(s)$ in $\mathbb{C}^{+}$. The computation of $G_{a}(s)$ is achieved by solving a Lyapunov equation of order $n_{b}$.

These two basic factorizations are then performed successively to get the inner-outer and spectral factorizations as explained in Section V.

\section{Outline of the paper}

The paper is organized as follows. In Section II we recall some results that make the connection between the SmithMcMillan invariants and the minimal indices of a rational matrix $G(s)$ on one side, and the Kronecker canonical form of the system pencil $\mathcal{S}_{G}(s)$ and of the pole pencil associated with a particular generalized state-space representation of $G(s)$ on the other side. In Section III we derive two special orthogonal decompositions of $\mathcal{S}_{G}(s)$ which exhibit the relevant Kronecker structure. These two spectral decompositions correspond to the two steps of the factorization process and are the key of our approach. Section IV contains the main results of the paper. There we present the two basic factorizations described above and for each factorization we provide explicit state-space formulas for the factors. We discuss the spectral and inner-outer factorizations in Section $\mathrm{V}$ and give an application to the computation of a generalized Moore-Penrose pseudoinverse in Section VI. In Section VII we give a detailed discussion of various numerical aspects of the problem and in Section VIII we present several numerical examples. We summarize the main aspects of the proposed approach by some conclusions that give insight into the nature of the considered factorization problems.

\section{Preliminaries}

Let $G(s)$ be an arbitrary (possibly improper) real rational matrix. Throughout the paper we use the following notation for the structural elements of $G(s): p \times m$ are its dimensions, $r$ stands for the rank over rational matrices, $n$ denotes the McMillan degree (which equals the number of poles including infinity and counting multiplicities), $n_{z}=n_{b}+n_{g}$ is the number of zeros (including infinity and counting multiplicities), where $n_{g}$ is the number of "good" zeros in $\overline{\mathbb{C}}^{-}$and $n_{b}$ is the number of "bad" zeros in $\mathbb{C}^{+}$, $\mathcal{Z}(G(s))$ denotes the union of zeros (with multiplicities), $n_{\ell}$ is the sum of degrees of any minimal basis of the left null space (these degrees are called left minimal indices), $n_{r}$ is the sum of degrees of any minimal basis of the right null space (these degrees are called right minimal indices). For precise definitions of all these structural elements see [15], [34], [44]. For a rational matrix there is an interesting relation [44] among its structural elements that will be insightful for the problems treated in the sequel:

$$
n=n_{z}+n_{r}+n_{\ell}
$$

It is well known that any rational matrix $G(s)$ (even improper or polynomial) has a descriptor realization

$$
G(s)=\left[\begin{array}{c|c}
A-s E & B \\
\hline C & D
\end{array}\right]:=C(s E-A)^{-1} B+D,
$$


where the so called pole pencil $A-s E$ is regular, i.e., it is square and $\operatorname{det}(A-s E) \not \equiv 0$. A pencil that is not regular is called singular. By $\Lambda(A-s E)$ we shall denote the union of generalized eigenvalues of an arbitrary (possibly singular) pencil $A-s E$ (finite and infinite, multiplicities counting).

The descriptor representation (8) of $G$ is called minimal if the dimension $k$ of the square matrices $E$ and $A$ is as small as possible. Well known criteria for minimality of a descriptor realization are (see for example [45]):

$$
\begin{aligned}
& \operatorname{rank}\left[\begin{array}{cc}
A-s E & B
\end{array}\right]=k, \quad \forall s \in \mathbb{C}, \\
& \operatorname{rank}\left[\begin{array}{cc}
E & B
\end{array}\right]=k, \\
& \operatorname{rank}\left[\begin{array}{c}
A-s E \\
C
\end{array}\right]=k, \quad \forall s \in \mathbb{C}, \\
& \operatorname{rank}\left[\begin{array}{c}
E \\
C
\end{array}\right]=k, \\
& A \operatorname{ker}(E) \subseteq \operatorname{Im}(E) .
\end{aligned}
$$

The conditions of minimality (i)-(v) are usually known as finite and infinite controllability, finite and infinite observability, and absence of nondynamic modes, respectively. We call weak minimal a realization that satisfies only (i)(iv). As we shall discuss later on, weak minimal realizations play an important role in numerical computations. Notice that for standard realizations (with $E$ invertible) minimality coincides with weak minimality.

For a minimal descriptor realization (8) of order $k$ we have $k=n+\kappa$, where $\kappa$ is the number of infinite elementary divisors and $n$ is the McMillan degree of $G(s)$ [44]. For a weak minimal realization we have $n=\operatorname{rank}(E)$. We make here the cautionary remark that for a pencil $A-s E$ the partial multiplicities of its infinite generalized eigenvalues are one in excess of the orders of its infinite zeros. For a careful discussion of poles and zeros at infinity see [44].

With a particular realization (8) of $G(s)$ we associate also the system pencil

$$
\mathcal{S}_{G}(s)=\left[\begin{array}{cc}
A-s E & B \\
C & D
\end{array}\right] .
$$

The pole pencil and the system pencil play a fundamental role as their Weierstrass and Kronecker canonical forms (see [16], [37]), respectively, are intimately related to the structural elements of the rational matrix as described in the following Theorem combined from [34] and [44].

Theorem II.1: Let $G(s)$ be an arbitrary rational matrix with a weak minimal realization (8) satisfying (i)-(iv) in (9).

1. Finite poles. The finite poles of $G(s)$ are the finite generalized eigenvalues of the pole pencil $A-s E$. The orders of the finite poles of $G(s)$ are pairwise equal to the multiplicities of the finite generalized eigenvalues of the pole pencil $A-s E$.

2. Infinite poles. The orders of the infinite poles of $G(s)$ are pairwise equal to the multiplicities of the infinite generalized eigenvalues of the pole pencil $A-s E$ minus 1 .
3. Finite zeros The finite zeros of $G(s)$ are the finite generalized eigenvalues of the system pencil $\mathcal{S}_{G}(s)$. The orders of the finite zeros of $G(s)$ are pairwise equal to the multiplicities of the finite generalized eigenvalues of the system pencil $\mathcal{S}_{G}(s)$.

4. Infinite zeros. The orders of the infinite zeros of $G(s)$ are pairwise equal to the multiplicities of the infinite generalized eigenvalues of the system pencil $\mathcal{S}_{G}(s)$ minus 1 .

5. Left minimal indices. The minimal indices to the left of $G(s)$ are pairwise equal to the elementary left Kronecker indices of the system pencil $\mathcal{S}_{G}(s)$.

6. Right minimal indices. The minimal indices to the right of $G(s)$ are pairwise equal to the elementary right Kronecker indices of the system pencil $\mathcal{S}_{G}(s)$.

\section{SPECTRAL DECOMPOSITIONS OF THE SYSTEM PENCIL}

In this section we give two spectral decompositions of the system pencil $\mathcal{S}_{G}(s)$ which correspond to the two basic factorizations described in Section I-C. Although these two results could have been presented jointly, we preferred to state them separately for the sake of the clarity. We start with a spectral decomposition that outlines in an appropriate form the left Kronecker indices of $\mathcal{S}_{G}(s)$.

Theorem III.1: Let $G(s)$ be a $p \times m$ real rational matrix of McMillan degree $n$, of rank $r$, having $n_{z}$ zeros, and the sums of minimal indices to the left and right $n_{\ell}$ and $n_{r}$, respectively. Then there exist a $k$-dimensional weak minimal realization (8) of $G(s)$ satisfying (i)-(iv) in (9), and two orthogonal matrices $Q$ and $Z$ such that

$$
\begin{aligned}
& {\left[\begin{array}{cc}
I & O \\
O & Q^{T}
\end{array}\right]\left[\begin{array}{cc}
A-s E & B \\
\hdashline C & D
\end{array}\right] Z=} \\
& \underbrace{\left[\begin{array}{cccc}
A_{r z}-s E_{r z} & B_{1}-s F_{1} & B_{2}-s F_{2} & B_{3}-s F_{3} \\
O & A_{\ell}-s E_{\ell} & B_{\ell} & B_{\ell n}-s F_{\ell n} \\
O & O & O & B_{n} \\
\hdashline & C_{\ell 1} & D_{\ell} & D_{1} \\
\hdashline & C_{\ell 2} & O & D_{2}
\end{array}\right] \begin{array}{l}
\} n_{r}+n_{z} \\
n_{\ell} \\
\} k-n \\
\} r \\
\} p-r
\end{array}}_{\begin{array}{cccc}
n_{r}+n_{z}+ \\
+m-r
\end{array}} \underbrace{}_{k-n}
\end{aligned}
$$

where

(a) $\mathcal{Z}(G(s))=\Lambda\left(A_{r z}-s E_{r z}\right)$ and $A_{r z}-s E_{r z}$ is surjective for all $s \notin \mathcal{Z}(G(s))$.

(b) $E_{\ell}, D_{\ell}, B_{n}$ are invertible and

$$
\begin{aligned}
& \text { (bi) } \operatorname{rank}\left[A_{\ell}-s E_{\ell} B_{\ell}\right]=n_{\ell}, \quad \forall s \in \mathbb{C}, \\
& \text { (bii) } \operatorname{rank}\left[\begin{array}{c}
A_{\ell}-B_{\ell} D_{\ell}^{-1} C_{\ell 1}-s E_{\ell} \\
C_{\ell 2}
\end{array}\right]=n_{\ell}, \forall s \in \mathbb{C} .
\end{aligned}
$$

Proof: We give here a constructive proof which serves simultaneously as a computational procedure to determine the appropriate minimal realization of $G(s)$ and the orthogonal matrices $Q$ and $Z$. We start with an arbitrary 
weak minimal realization (8) of $G(s)$ satisfying (i)-(iv) in $(9)$, and let $\mathcal{S}_{G}(s)$ be the associated system pencil.

Step 1. Compute orthogonal matrices $U_{1}$ and $V_{1}$ such that

$$
U_{1} E V_{1}=\left[\begin{array}{cc}
E_{11}^{(1)} & O \\
O & O
\end{array}\right]
$$

where $E_{11}^{(1)}$ is invertible. Define

$$
\begin{aligned}
& \mathcal{S}_{1}(s):=\operatorname{diag}\left(U_{1}, I\right) \mathcal{S}_{G}(s) \operatorname{diag}\left(V_{1}, I\right) \\
= & {\left[\begin{array}{ccc}
A_{11}^{(1)}-s E_{11}^{(1)} & A_{12}^{(1)} & B_{1}^{(1)} \\
A_{21}^{(1)} & A_{22}^{(1)} & B_{2}^{(1)} \\
\hdashline C_{1}^{(1)} & C_{2}^{(1)} & D
\end{array}\right] \begin{array}{l}
\} n \\
\} k-n . \\
\} p
\end{array} }
\end{aligned}
$$

From (ii) in (9) we have

$$
\text { (1i) } \operatorname{rank}\left[\begin{array}{lll}
A_{21}^{(1)} & A_{22}^{(1)} & B_{2}^{(1)}
\end{array}\right]=\operatorname{rank} B_{2}^{(1)}=k-n .
$$

Step 2. Compute orthogonal $Z_{1}$ such that

$$
\left[\begin{array}{lll}
A_{21}^{(1)} & A_{22}^{(1)} & B_{2}^{(1)}
\end{array}\right] Z_{1}=\left[\begin{array}{ll}
O & B_{n}
\end{array}\right]
$$

where $B_{n}$ is invertible. This is always possible due to (1i). Define

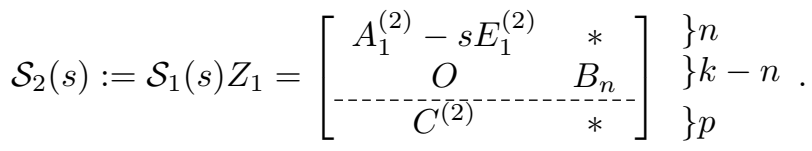

Step 3. Compute orthogonal $Z_{2}$ such that

$$
C^{(2)} Z_{2}=\left[\begin{array}{ll}
O & C_{2}^{(3)}
\end{array}\right]
$$

with $C_{2}^{(3)}$ injective and define

$$
\begin{gathered}
\mathcal{S}_{3}(s):=\mathcal{S}_{2}(s) \operatorname{diag}\left(Z_{2}, I\right) \\
=\left[\begin{array}{ccc}
A_{11}^{(3)}-s E_{11}^{(3)} & A_{12}^{(3)}-s E_{12}^{(3)} & * \\
O & O & B_{n} \\
\hdashline O & C_{2}^{(3)} & *
\end{array}\right] \begin{array}{l}
\} n \\
\} k-n . \\
\} p
\end{array}
\end{gathered}
$$

From (i) and (ii) in (9) we have

$$
\begin{aligned}
& \text { (3i) } \operatorname{rank}\left[\begin{array}{cc}
E_{11}^{(3)} & E_{12}^{(3)}
\end{array}\right]=n, \\
& \text { (3ii) } \operatorname{rank}\left[A_{11}^{(3)}-s E_{11}^{(3)} A_{12}^{(3)}-s E_{12}^{(3)}\right]=n, \forall s \in \mathbb{C},
\end{aligned}
$$

and the resulting pencil $A_{11}^{(3)}-s E_{11}^{(3)}$ has the following properties:

$$
\text { (3iii) } \mathcal{Z}(G(s))=\Lambda\left(A_{11}^{(3)}-s E_{11}^{(3)}\right) \text {; }
$$

(3iv) The entire right Kronecker structure of $\mathcal{S}_{G}(s)$ is contained in $\Lambda\left(A_{11}^{(3)}-s E_{11}^{(3)}\right)$, i.e., their right Kronecker indices are pairwise equal;

(3v) All nonzero Kronecker indices of $\mathcal{S}_{G}(s)$ are isomorphic with the Kronecker indices of $A_{11}^{(3)}-s E_{11}^{(3)}$ in the following sense: to each nonzero left Kronecker index $\eta_{i}$ of $\mathcal{S}_{G}(s)$ corresponds a left Kronecker index $\eta_{i}-1$ of $A_{11}^{(3)}-s E_{11}^{(3)}$.

Properties (3iii)-(3v) follow by extending Lemma 4.1 in [40] to the case of an improper rational matrix. As the extension is straightforward, we do not give the detailed proof here.

Step 4. Compute orthogonal $U_{3}$ and $Z_{3}$ to reduce the subpencil $A_{11}^{(3)}-s E_{11}^{(3)}$ to a Kronecker-like form [37]

$$
\left.U_{3}\left(A_{11}^{(3)}-s E_{11}^{(3)}\right) Z_{3}=\left[\begin{array}{c}
A_{z r}-s E_{r z} * \\
O
\end{array} \bar{A}_{\ell}-s \bar{E}_{\ell}\right]\right\} \begin{aligned}
& \} n_{r}+n_{z} \\
& n_{\ell}
\end{aligned}
$$

where it follows from (3iii)-(3iv) that $A_{r z}-s E_{r z}$ contains the right Kronecker structure of $\mathcal{S}_{G}(s)$ and the zeros of $G(s)$, and thus satisfies (a). Further,

(4i) $\bar{A}_{\ell}-s \bar{E}_{\ell}$ is injective, $\forall s \in \mathbb{C}$;

(4ii) $\bar{E}_{\ell}$ is injective,

and from $(3 \mathrm{v})$ we have that all nonzero Kronecker indices of $\mathcal{S}_{G}(s)$ are isomorphic with the Kronecker indices of $\bar{A}_{\ell}-s \bar{E}_{\ell}$ as explained at (3v). Define

$$
\begin{gathered}
\mathcal{S}_{4}(s):=\operatorname{diag}\left(U_{3}, I\right) \mathcal{S}_{3}(s) \operatorname{diag}\left(Z_{3}, I\right) \\
=\left[\begin{array}{cccc}
A_{r z}-s E_{r z} & * & * & * \\
O & \bar{A}_{\ell}-s \bar{E}_{\ell} \bar{B}_{\ell}-s \bar{F}_{\ell} & * \\
O & O & O & B_{n} \\
\hdashline O & O_{\times} & C_{2}^{(\overline{3})^{-}} & *
\end{array}\right] \begin{array}{l}
\} n_{r}+n_{z} \\
\} n_{\ell} \\
\} k-n \\
\} p
\end{array} .
\end{gathered}
$$

From (3i) and (3ii) we get

$$
\begin{aligned}
& \text { (4iii) } \operatorname{rank}\left[\begin{array}{ll}
\bar{E}_{\ell} & \bar{F}_{\ell}
\end{array}\right]=n_{\ell}, \\
& \text { (4iv) } \operatorname{rank}\left[\begin{array}{ll}
\bar{A}_{\ell}-s \bar{E}_{\ell} & \bar{B}_{\ell}-s \bar{F}_{\ell}
\end{array}\right]=n_{\ell}, \quad \forall s \in \mathbb{C},
\end{aligned}
$$

and since $C_{2}^{(3)}$ is injective we have also from (4i) and (4ii) that

$$
\begin{aligned}
& (\mathbf{4 v})\left[\begin{array}{cc}
\bar{A}_{\ell}-s \bar{E}_{\ell} & \bar{B}_{\ell}-s \bar{F}_{\ell} \\
O & C_{2}^{(3)}
\end{array}\right] \text { is injective, } \forall s \in \mathbb{C}, \\
& \left(\mathbf{4 v i )}\left[\begin{array}{cc}
\bar{E}_{\ell} & \bar{F}_{\ell} \\
O & C_{2}^{(3)}
\end{array}\right]\right. \text { is injective. }
\end{aligned}
$$

Step 5. Compute orthogonal $Z_{4}$ such that

$$
\left[\begin{array}{cc}
\bar{E}_{\ell} & \bar{F}_{\ell} \\
O_{\times} & C_{2}^{(3)}
\end{array}\right] Z_{4}=\left[\begin{array}{cc}
E_{\ell} & O \\
\bar{C}_{\ell} & \bar{D}_{\ell}
\end{array}\right],
$$

with $E_{\ell}$ invertible, which is possible due to (4iii). It follows from (4vi) that $\bar{D}_{\ell}$ is injective. Compute orthogonal $Q$ such that

$$
Q^{T}\left[\begin{array}{ll}
\bar{C}_{\ell} & \bar{D}_{\ell}
\end{array}\right]=\left[\begin{array}{ll}
C_{\ell 1} & D_{\ell} \\
C_{\ell 2} & O
\end{array}\right],
$$

with $D_{\ell}$ invertible. Define

$$
\mathcal{S}_{5}(s):=\operatorname{diag}\left(I, Q^{T}\right) \mathcal{S}_{4}(s) \operatorname{diag}\left(I, Z_{4}, I\right)=
$$




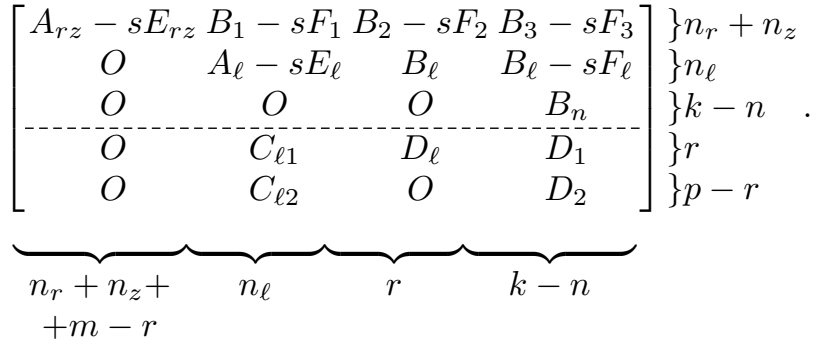

From (4iv) it follows that (bi) is satisfied and from $(4 \mathrm{v})$ and straightforward manipulations we get that (bii) is satisfied as well.

Step 6. Define

$$
\begin{gathered}
U=U_{3} U_{1}, V=V_{1} \\
Z=\operatorname{diag}\left(V_{1}^{T}, I\right) Z_{1} \operatorname{diag}\left(Z_{2}, I\right) \operatorname{diag}\left(Z_{3}, I\right) \operatorname{diag}\left(I, Z_{4}, I\right) .
\end{gathered}
$$

Overall, we have determined matrices $U, V, Q$ and $Z$ such that

$$
\left[\begin{array}{cc}
I & O \\
O & Q^{T}
\end{array}\right]\left[\begin{array}{cc}
U(A-s E) V & U B \\
\hdashline C & D
\end{array}\right] Z=\mathcal{S}_{5}(s) .
$$

We "absorb" $U$ and $V$ as generalized state-space transformations in the initial realization (8), and obtain a new realization denoted also (8). The new realization (8) and the matrices $Q$ and $Z$ satisfy all the conditions in the statement of the Theorem and this ends the proof.

We assume now that $G(s)$ has no left minimal indices, i.e., it is surjective. As we shall see further, this is always possible after we have performed the first step of the factorization process. The theorem below provides a special spectral decomposition of the system pencil $\mathcal{S}_{G}(s)$ that is key to understanding the conditions for dislocating the zeros in $\mathbb{C}_{+}$of $G(s)$.

Theorem III.2: Let $G(s)$ be a $p \times m$ real rational matrix of McMillan degree $n$, of rank $p$ (i.e., $G(s)$ is surjective), having the sum of minimal indices to the right $n_{r}$, and a number of $n_{z}=n_{b}+n_{g}$ zeros, where $n_{b}$ and $n_{g}$ are the numbers of zeros in $\mathbb{C}^{+}$and $\overline{\mathbb{C}}^{-}$, respectively. Then there exists a $k$-dimensional weak minimal realization (8) of $G(s)$ satisfying conditions (i)-(iv) in (9), and an orthogonal matrix $Z$ such that

$$
\begin{aligned}
& {\left[\begin{array}{cc}
A-s E & B \\
\hdashline C & D
\end{array}\right] Z=} \\
& {\left[\begin{array}{cccc}
A_{r g}-s E_{r g} & B_{1}-s F_{1} & B_{2}-s F_{2} & B_{3}-s F_{3} \\
O & A_{b}-s E_{b} & B_{b} & B_{b n}-s F_{b n} \\
O & O & O & B_{n} \\
\hdashline O & C_{b} & D_{b} & D_{1}^{-}
\end{array}\right] \begin{array}{l}
\} n_{r}+n_{g} \\
\} n_{b} \\
\} k-n \\
\} p
\end{array}} \\
& \underbrace{}_{n_{r}+n_{b}+} \overbrace{n_{b}} \overbrace{p} \overbrace{k-n}^{1} \\
& +m-p
\end{aligned}
$$

where

(a) $\mathcal{Z}(G(s)) \cap \overline{\mathbb{C}}^{-}=\Lambda\left(A_{r g}-s E_{r g}\right)$ and $A_{r g}-s E_{r g}$ is surjective for all $s \notin \mathcal{Z}(G(s)) \cap \overline{\mathbb{C}^{-}}$, (b) $E_{b}, D_{b}, B_{n}$ are invertible and

(bi) $\Lambda\left(A_{b}-B_{b} D_{b}^{-1} C_{b}-s E_{b}\right)=\mathcal{Z}(G(s)) \cap \mathbb{C}_{+}$,

(bii) $\operatorname{rank}\left[\begin{array}{cc}A_{b}-s E_{b} & B_{b}\end{array}\right]=n_{b}, \quad \forall s \in \mathbb{C}$.

Proof: The proof is very similar to the proof of Theorem III.1. We note first that since $G(s)$ is surjective Theorem II.1 shows that the system pencil $\mathcal{S}_{G}(s)$ is surjective as well.

Steps 1.-3. Perform Steps 1-3 as in the proof of Theorem III. 1 to obtain

$\mathcal{S}_{3}(s):=\left[\begin{array}{ccc}A_{11}^{(3)}-s E_{11}^{(3)} & A_{12}^{(3)}-s E_{12}^{(3)} & * \\ O & O & B_{n} \\ \hdashline O & C_{2}^{(3)} & *\end{array}\right] \begin{aligned} & \} n \\ & \} p-n\end{aligned}$

satisfying (3i)-(3iv). Since $\mathcal{S}_{G}(s)$ is surjective it follows from (17) that $C_{2}^{(3)}$ is actually invertible.

Step 4. Compute orthogonal $U_{3}$ and $Z_{3}$ to reduce the subpencil $A_{11}^{(3)}-s E_{11}^{(3)}$ to a Kronecker-like form [37]

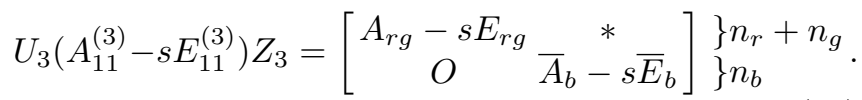

It follows from (3iii)-(3iv) that the pencil $A_{r g}-s E_{r g}$ contains the right Kronecker structure of $\mathcal{S}_{G}(s)$ and all zeros of $G(s)$ in $\overline{\mathbb{C}}^{-}$and thus satisfies (a). Further, $\bar{A}_{b}-$ $s \bar{E}_{b}$ is regular and

$$
\Lambda\left(\bar{A}_{b}-s \bar{E}_{b}\right)=\mathcal{Z}(G(s)) \cap \mathbb{C}^{+},
$$

and thus $\bar{E}_{b}$ is invertible. Define

$$
\begin{gathered}
\mathcal{S}_{4}(s):=\operatorname{diag}\left(U_{3}, I\right) \mathcal{S}_{3}(s) \operatorname{diag}\left(Z_{3}, I\right) \\
=\left[\begin{array}{cccc}
A_{r g}-s E_{r g} & * & * & * \\
O & \bar{A}_{b}-s \bar{E}_{b} & \bar{B}_{b}-s \bar{F}_{b} & * \\
O & O & O & B_{n} \\
\hdashline O & O_{x} & \bar{D}_{b} & *
\end{array}\right] \begin{array}{l}
\} n_{r}+n_{g} \\
\} \\
n_{b} \\
\} p-n
\end{array}
\end{gathered}
$$

where $\bar{D}_{b}:=C_{2}^{(3)}$. From (3ii) we get

$$
\text { (4ii) } \operatorname{rank}\left[\bar{A}_{b}-s \bar{E}_{b} \quad \bar{B}_{b}-s \bar{F}_{b}\right]=n_{b}, \quad \forall s \in \mathbb{C}
$$

and since $\bar{D}_{b}$ and $\bar{E}_{b}$ are invertible we have also that

$$
\text { (4iii) }\left[\begin{array}{cc}
\bar{E}_{b} & \bar{F}_{b} \\
O & \bar{D}_{b}
\end{array}\right] \text { is invertible. }
$$

Step 5. Compute orthogonal $Z_{4}$ such that

$$
\left[\begin{array}{ll}
\bar{E}_{b} & \bar{F}_{b}
\end{array}\right] Z_{4}=\left[\begin{array}{ll}
E_{b} & O
\end{array}\right]
$$

with $E_{b}$ invertible, which is possible since $\bar{E}_{b}$ is invertible. Define

$$
\mathcal{S}_{5}(s):=\mathcal{S}_{4}(s) \operatorname{diag}\left(I, Z_{4}, I\right)=
$$




$$
\begin{aligned}
& \left.\left[A_{r g}-s E_{r g} B_{1}-s F_{1} B_{2}-s F_{2} B_{3}-s F_{3}\right]\right\} n_{r}+n_{g} \\
& \left.\left[\begin{array}{cccc}
O & A_{b}-s E_{b} & B_{b} & B_{b n}-s F_{b n} \\
O & O & O & B_{n} \\
\hdashline O & C_{b} & D_{b} & D_{1}
\end{array}\right]\right\} \begin{array}{l}
\} n_{b} \\
\} k-n
\end{array} \\
& \underbrace{\ln }_{n_{r}+n_{g}+} \overbrace{n_{b}} \overbrace{p} \overbrace{k-n} \\
& +m-p
\end{aligned}
$$

From (4iii) it follows that $D_{b}$ is invertible and (bi) follows immediately from (4i) as $\Lambda\left(A_{b}-B_{b} D_{b}^{-1} C_{b}-s E_{b}\right)=$ $\Lambda\left(\bar{A}_{b}-s \bar{E}_{b}\right)$. Finally, (bii) is a consequence of (4ii).

Step 6. Define

$$
\begin{gathered}
U=U_{3} U_{1}, \quad V=V_{1}, \\
Z=\operatorname{diag}\left(V_{1}^{T}, I\right) Z_{1} \operatorname{diag}\left(Z_{2}, I\right) \operatorname{diag}\left(Z_{3}, I\right) \operatorname{diag}\left(I, Z_{4}, I\right) .
\end{gathered}
$$

Overall we have determined matrices $U, V$, and $Z$ such that

$$
\left[\begin{array}{cc}
U(A-s E) V & B U \\
\hdashline \bar{C} & \bar{D}
\end{array}\right] Z=\mathcal{S}_{5}(s)
$$

We "absorb" $U$ and $V$ as generalized state-space transformations in the initial realization (8), and obtain a new realization denoted also (8). The new realization (8) and the matrix $Z$ satisfy all the conditions in the statement of the theorem.

\section{The BASIC FACTORIZATION STEPS}

In this section we describe the factorization steps described in Section I-C. Throughout this section $n_{b}$ and $n_{g}$ denote the number of zeros of $G(s)$ in $\mathbb{C}^{+}$and $\overline{\mathbb{C}}^{-}$, respectively. We start with the solution to the row compression problem.

\section{A. Row compression by all-pass factors}

Theorem IV.1: Let $G(s)$ be a real rational matrix with a weak minimal realization (8) as in Theorem III.1 and let $Q$ and $Z$ be orthogonal matrices for which (11) holds. Then we have:

1. The continuous-time algebraic Riccati equation

$$
\begin{array}{r}
A_{\ell}^{T} X E_{\ell}+E_{\ell}^{T} X A_{\ell}-\left(E_{\ell}^{T} X B_{\ell}+C_{\ell 1}^{T} D_{\ell}\right) \times \\
\times\left(D_{\ell}^{T} D_{\ell}\right)^{-1}\left(B_{\ell}^{T} X E_{\ell}+D_{\ell}^{T} C_{\ell 1}\right)+C_{\ell}^{T} C_{\ell}=0
\end{array}
$$

has a stabilizing symmetric positive definite solution $X_{s}$ such that $\Lambda\left(A_{\ell}+B_{\ell} F_{s}-s E_{\ell}\right) \subset \mathbb{C}^{-}$, where

$$
F_{s}:=-\left(D_{\ell}^{T} D_{\ell}\right)^{-1}\left(B_{\ell}^{T} X_{s} E_{\ell}+D_{\ell}^{T} C_{\ell 1}\right)
$$

is the stabilizing Riccati feedback and $C_{\ell}^{T}:=\left[\begin{array}{ll}C_{\ell 1}^{T} & C_{\ell 2}^{T}\end{array}\right]$.

2. Let

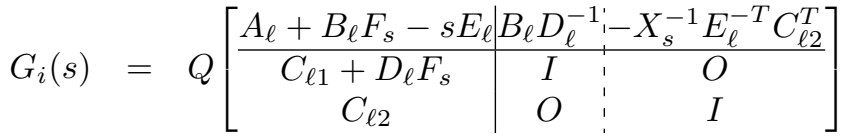

$$
\begin{aligned}
& =\left[\begin{array}{l:l}
G_{i 1}(s) & G_{i 2}(s)
\end{array}\right]
\end{aligned}
$$

and

$$
G_{o}(s)=\left[\begin{array}{c|c}
A-s E & B \\
\hline D_{\ell} H_{1} & D_{\ell} H_{2} \\
\hdashline O & O
\end{array}\right]=\left[\begin{array}{c}
G_{o 1}(s) \\
\hdashline O
\end{array}\right]
$$

where

$$
\left[\begin{array}{ll}
H_{1} & H_{2}
\end{array}\right]:=\left[\begin{array}{llll}
O & -F_{s} & I & O
\end{array}\right] Z^{T} .
$$

Then $G_{i}(s)$ is a $p \times p$ inner matrix, the realization (23) is minimal, $G(s)=G_{i}(s) G_{o}(s), G_{o 1}(s)$ has no left minimal indices, and $\mathcal{Z}\left(G_{o 1}(s)\right)$ has $n_{b}$ elements in $\mathbb{C}^{+}$.

Proof: 1. From (b) of Theorem III.1 we have that $E_{\ell}$ is invertible. From (21) we get after straightforward manipulations

$$
\begin{gathered}
\left(\widetilde{A}_{\ell}-B_{\ell} D_{\ell}^{-1} \widetilde{C}_{\ell 1}\right)^{T} X+X\left(\widetilde{A}_{\ell}-B_{\ell} D_{\ell}^{-1} \widetilde{C}_{\ell 1}\right) \\
-X B_{\ell}\left(D_{\ell}^{T} D_{\ell}\right)^{-1} B_{\ell}^{T} X+\widetilde{C}_{\ell 2}^{T} \widetilde{C}_{\ell 2}=0
\end{gathered}
$$

where $\widetilde{A}_{\ell}:=A_{\ell} E_{\ell}^{-1}, \widetilde{C}_{\ell 1}:=C_{\ell 1} E_{\ell}^{-1}, \widetilde{C}_{\ell 2}:=C_{\ell 2} E_{\ell}^{-1}$. Then (bi) and (bii) in Theorem III.1 show that the pair $\left(\widetilde{A}_{\ell}-B_{\ell} D_{\ell}^{-1} \widetilde{C}_{\ell 1}, B_{\ell}\right)$ is controllable and the pair $\left(C_{\ell 2}, \widetilde{A}_{\ell}-\right.$ $\left.B_{\ell} D_{\ell}^{-1} \widetilde{C}_{\ell 1}\right)$ is observable, and from Theorem 13.7 in [50] we conclude that the algebraic Riccati equation (26) has an invertible positive definite stabilizing solution $X_{s}$ which is the stabilizing solution to (21) as well.

2. Clearly $G_{i}(s)$ in $(23)$ has all poles in $\mathbb{C}^{-}$. To show that $G_{i}(s)$ is inner we can prove equivalently that its inverse $G_{i}^{-1}(s)$ is all-pass. Using (23) we obtain a realization of $G_{i}^{-1}(s)$ in the form

$$
\begin{gathered}
G_{i}^{-1}(s)=\left[\begin{array}{c|c}
A_{i}-s E_{i} \mid B_{i} \\
\hline C_{i} & D_{i}
\end{array}\right] Q^{T}=\left[\begin{array}{c|cc}
A_{i}-s E_{i} & B_{i 1} & B_{i 2} \\
\hline C_{i 1} & I & O \\
C_{i 2} & O & I
\end{array}\right] Q^{T} \\
\stackrel{(23)}{=}\left[\begin{array}{c|c}
A_{\ell}-B_{\ell} D_{\ell}^{-1} C_{\ell 1}+X_{s}^{-1} E_{\ell}^{-T} C_{\ell 2}^{T} C_{\ell 2}-s E_{\ell} & B_{\ell} D_{\ell}^{-1} \\
\hline-\left(C_{\ell 1}+D_{\ell} F_{s}\right) & I \\
-C_{\ell 2} & O \\
\frac{-X_{s}^{-1} E_{\ell}^{-T} C_{\ell 2}^{T}}{O} \\
I
\end{array}\right] Q^{T} .
\end{gathered}
$$

By direct substitution we check that the following equations hold

$$
\begin{aligned}
A_{i}^{T} X_{s} E_{i}+E_{i}^{T} X_{s} A_{i}-C_{i}^{T} C_{i} & =0 \\
C_{i}-D_{i} B_{i}^{T} X_{s} E_{i} & =0 \\
D_{i}^{T} D_{i} & =I .
\end{aligned}
$$

This shows that $G_{i}(s)^{-1} Q$ is all-pass (see [50]) and since $Q$ is orthogonal we finally get that $G_{i}(s)$ is inner. Moreover, since the pair $\left(A_{\ell}-s E_{\ell}, B_{\ell}\right)$ is controllable it is easy to see that the realization (27) is controllable as well. Further, as $X_{s}$ is invertible we get from (28) that the realization (27) is also observable and thus minimal. 
Before proceeding with the rest of the proof we make several preparations. With (27) we get

$$
\left[O I W_{n}-B_{i 1}-B_{i 2}\right]\left[\begin{array}{ccc}
A_{r z}-s E_{r z} & B_{1}-s F_{1} & B_{2}-s F_{2} \\
O & A_{\ell}-s E_{\ell} & B_{\ell} \\
O & O & O \\
O & C_{\ell 1} & D_{\ell} \\
O & C_{\ell 2} & O
\end{array}\right.
$$

$$
\left.\begin{array}{c}
B_{3}-s F_{3} \\
B_{\ell n}-s F_{\ell n} \\
B_{n} \\
D_{1} \\
D_{2}
\end{array}\right]=\left(A_{i}-s E_{i}\right)\left[O I O E_{i}^{-1} F_{\ell n}\right]
$$

where $W_{n}:=\left(A_{i} E_{i}^{-1} F_{\ell n}+B_{i 1} D_{1}+B_{i 2} D_{2}-B_{\ell n}\right) B_{n}^{-1}$ and the inverse of the $B_{n}$ exists due to (b) of Theorem III.1. Multiplying (29) to the right with $Z^{T}$ we get with (11)

$$
\left[W_{1}-B_{i}\right]\left[\begin{array}{cc}
A-s E & B \\
Q^{T} C & Q^{T} D
\end{array}\right]=\left(A_{i}-s E_{i}\right)\left[W_{2} O\right],
$$

where

$$
\begin{gathered}
W_{1}:=\left[\begin{array}{lll}
O & I & W_{n}
\end{array}\right] \\
{\left[\begin{array}{ll}
W_{2} & O
\end{array}\right]:=\left[\begin{array}{llll}
O & I & O & E_{i}^{-1} F_{\ell n}
\end{array}\right] Z^{T}}
\end{gathered}
$$

and the "zero" pattern of the left-hand side of (32) follows from (30) and the invertibility of $E_{i}=E_{\ell}$.

We show now that $G(s)=G_{i}(s) G_{o}(s)$. To this end we use $(27)$ to write a state-space representation of $G_{i}^{-1}(s) G(s)$ in the form

$$
G_{i}^{-1}(s) G(s)=\left[\begin{array}{cc|c}
A_{i}-s E_{i} & B_{i} Q^{T} C & B_{i} Q^{T} D \\
O & A-s E & B \\
\hline C_{i} & Q^{T} C & Q^{T} D
\end{array}\right]
$$

and performing an equivalence transformation on (33) we get

$$
\begin{aligned}
G_{i}^{-1}(s) G(s) & =\left[\begin{array}{cc|c}
A_{i}-s E_{i} & O & O \\
O & A-s E & B \\
\hline C_{i} & C_{i} W_{2}+Q^{T} C & Q^{T} D
\end{array}\right] \\
& =\left[\begin{array}{c|c}
A-s E & B \\
\hline C_{i} W_{2}+Q^{T} C & Q^{T} D
\end{array}\right]
\end{aligned}
$$

where we have used (30). To prove that $G_{i}^{-1}(s) G(s)$ given by $(34)$ and $G_{o}(s)$ given by $(24)$ are equal we show that the system pencils associated with these two realizations are related by a transformation that does not change the transfer function matrix. We have

$$
\begin{aligned}
\mathcal{S}_{G_{i}^{-1} G}(s) & \stackrel{(34)}{=}\left[\begin{array}{cc}
A-s E & B \\
C_{i} W_{2}+Q^{T} C & Q^{T} D
\end{array}\right] \\
& =\left[\begin{array}{cc}
I & O \\
O & Q^{T}
\end{array}\right]\left[\begin{array}{cc}
A-s E & B \\
C & D
\end{array}\right]+\left[\begin{array}{c}
O \\
C_{i}
\end{array}\right]\left[W_{2} O\right]
\end{aligned}
$$

from which it follows with (27), (32), and (11) that

$$
\begin{aligned}
& \mathcal{S}_{G_{i}^{-1} G}(s) Z \\
& =\left[\begin{array}{cccc}
A_{r z}-s E_{r z} & B_{1}-s F_{1} & B_{2}-s F_{2} & B_{3}-s F_{3} \\
O & A_{\ell}-s E_{\ell} & B_{\ell} & B_{\ell n}-s F_{\ell n} \\
O & O & O & B_{n} \\
\hdashline O & -D_{\ell} F_{s} & D_{\ell} & D_{1}+C_{i 1} E_{i}^{-1} F_{\ell n} \\
O & O & O & D_{2}+C_{i 2} E_{i}^{-1} F_{\ell n}
\end{array}\right] .
\end{aligned}
$$

On the other hand, we get from (24) and (25)

$$
\mathcal{S}_{G_{o}}(s) Z=\left[\begin{array}{cccc}
A_{r z}-s E_{r z} & B_{1}-s F_{1} & B_{2}-s F_{2} & B_{3}-s F_{3} \\
O & A_{\ell}-s E_{\ell} & B_{\ell} & B_{\ell n}-s F_{\ell n} \\
O & O & O & B_{n} \\
\hdashline O & -D_{\ell} F_{s} & D_{\ell} & O \\
O & O & O & O
\end{array}\right] .
$$

Comparing (36) with (37), we get that

$$
\mathcal{S}_{G_{o}}(s)=V \mathcal{S}_{G_{i}^{-1} G}(s)
$$

where

$$
V=\left[\begin{array}{ccc:cc}
I & O & O & O & O \\
O & I & O & O & O \\
O & O & I & O & O \\
\hdashline O & O & -\left(D_{1}+C_{i 1} E_{i}^{-1} F_{\ell}\right) B_{n}^{-1} & I & O \\
O & O & -\left(D_{2}+C_{i 2} E_{i}^{-1} F_{\ell}\right) B_{n}^{-1} & O & I
\end{array}\right]
$$

The partition in (39) shows that $V$ is a Rosenbrock type transformation relating the system pencils $\mathcal{S}_{G_{o}}(s)$ and $\mathcal{S}_{G_{i}^{-1} G}(s)$ as in (38). Hence the transfer function matrices corresponding to these system pencils are equal, i.e., $G_{i}^{-1}(s) G(s)=G_{o}(s)$.

It only remains to show that $G_{o 1}(s)$ has no left minimal indices and $\mathcal{Z}\left(G_{o}(s)\right)$ has $n_{b}$ elements in $\mathbb{C}^{+}$. It is easy to see from $(37)$ and $(24)$ that the system pencil $\mathcal{S}_{G_{o 1}}(s)$ is strictly equivalent to

$$
\left[\begin{array}{cccc}
A_{r z}-s E_{r z} & * & * & * \\
O & A_{\ell}+B_{\ell} F_{s}-s E_{\ell} & B_{\ell} & * \\
O & O & O & B_{n} \\
\hdashline O & O & D_{\ell} & O
\end{array}\right]
$$

and from (a) of Theorem III.1 we conclude that (40) has no left Kronecker indices and its zeros are given by $\Lambda\left(A_{r z}-\right.$ $\left.s E_{r z}\right) \cup \Lambda\left(A_{\ell}+B_{\ell} F_{s}-s E_{\ell}\right)$. We have $\Lambda\left(A_{\ell}+B_{\ell} F_{s}-\right.$ $\left.s E_{\ell}\right) \subset \mathbb{C}^{-}, \Lambda\left(A_{r z}-s E_{r z}\right)=\mathcal{Z}(G(s))$ has $n_{b}$ elements in $\mathbb{C}^{+}$and thus the pencil $S_{G_{o 1}(s)}$ has no left Kronecker indices and has $n_{b}$ zeros in $\mathbb{C}^{+}$. Assume temporarily that the resulting realization $(24)$ is weak minimal. Then we have with Theorem II.1 that $\mathcal{Z}\left(G_{o}(s)\right)=\mathcal{Z}\left(\mathcal{S}_{G_{o}}(s)\right)=$ $\mathcal{Z}\left(\mathcal{S}_{G_{o 1}}(s)\right)=\Lambda\left(A_{r z}-s E_{r z}\right) \cup \Lambda\left(A_{\ell}+B_{\ell} F_{s}-s E_{\ell}\right)$ and thus $G_{o 1}(s)$ has $n_{b}$ zeros in $\mathbb{C}^{+}$while the absence of left minimal indices follows from the one-to-one correspondence among the left minimal indices of $G_{o 1}(s)$ and the left elementary Kronecker indices of $\mathcal{S}_{G_{o} 1}(s)$ (see point 5 of Theorem II.1).

Finally, we remove the assumption that the resulting realization (24) is weak minimal. Nonminimality may occur 
if in the product $G_{o}(s)=G_{i}(s)^{-1} G(s)$ a cancellation between a zero of $G_{i}(s)^{-1}$ and a pole of $G(s)$ takes place. This happens when $G(s)$ contains so-called free inner factors (see [18]). We show first the claim for the zeros. We have

$$
G(s)=G_{i}(s) G_{o}(s)=G_{i 1}(s) G_{o 1}(s)
$$

and it is easy to see from (23) that $G_{i 1}(s)$ is injective and has no zeros, i.e., it is injective for all $s$, and has all its poles in $\mathbb{C}^{-}$. It follows then from (41) and the SmithMcMillan form (or from a Laurent expansion around any point in $\mathbb{C}^{+}$) that $G(s)$ and $G_{o 1}(s)$ share the same zeros in $\mathbb{C}^{+}$(counting multiplicities). Thus $G_{01}(s)$ has $n_{b}$ zeros in $\mathbb{C}^{+}$. We show now the claim for the minimal indices. We note that if the system pencil $S_{G_{o 1}}(s)$ associated with an arbitrary realization (even nonminimal) of $G_{o 1}(s)$ is surjective (i.e., it has no left Kronecker structure) then $G_{o 1}(s)$ is surjective as well. As $\mathcal{S}_{G_{o 1}}(s)$ has no left Kronecker indices, it follows that $\mathcal{S}_{G_{o 1}}(s)$ is surjective and thus $G_{o 1}(s)$ has no left minimal indices.

Remark IV.2: The above theorem gives in fact a solution to the following problem: Given an arbitrary transfer matrix $G(s)$ determine an all-pass rational matrix $G_{a}(s)$ $\left(=G_{i}(s)^{-1}\right)$, of minimal McMillan degree that dislocates, by left multiplication, all minimal indices of $G(s)$ and makes them zero, while it introduces "good" zeros (in $\mathbb{C}^{-}$) instead. The resulting $G_{o}(s)=G_{a}(s) G(s)$ has the same structural elements as $G(s)$ excepting some additional zeros in $\mathbb{C}^{-}$and the minimal basis for the left null space which are built up from constant elements (polynomial degree 0 ). If $G(s)$ has only "good" zeros (in $\left.\overline{\mathbb{C}}^{+}\right)$then $G_{o}(s)$ is already a solution to the inner-outer factorization problem. Comparing our formulas for the Riccati equation and for the inner and outer factors with the formulas in [50] (on page 367 ), we see that our method has extracted from the original realization of $G(s)$ a proper subsystem

$$
G_{\ell}(s)=\left[\begin{array}{c|c}
A_{\ell}-s E_{\ell} & B_{\ell} \\
\hline C_{\ell 1} & D_{\ell} \\
C_{\ell 2} & O
\end{array}\right]
$$

which is left invertible and without zeros, and it has solved the corresponding inner-outer factorization problem for this subsystem. Then the solution to the original innerouter factorization problem for $G(s)$ follows immediately. Moreover, for extracting (42) from the original realization of $G(s)$ we have performed exclusively orthogonal transformations and the subsystem (42) is the smallest one possible.

We switch now to the problem of dislocating zeros.

\section{B. Dislocation of zeros by all-pass factors}

Theorem IV.3: Let $G(s)$ be a surjective real rational matrix with a weak minimal realization (8) as in Theorem III.2 and let $Z$ be an orthogonal matrix for which (15) holds. Then:

1. The Lyapunov equation

$$
\left(A_{b}-B_{b} D_{b}^{-1} C_{b}\right) Y E_{b}^{T}+E_{b} Y\left(A_{b}-B_{b} D_{b}^{-1} C_{b}\right)^{T}
$$

$$
-B_{b}\left(D_{b}^{T} D_{b}\right)^{-1} B_{b}^{T}=0
$$

has a unique symmetric positive definite solution such that $\Lambda\left(A_{b}+B_{b} F_{s}-s E_{b}\right) \subset \mathbb{C}^{-}$, where

$$
F_{s}:=-\left(D_{b}^{T} D_{b}\right)^{-1}\left(B_{b}^{T} E_{b}^{-T} Y^{-1}+D_{b}^{T} C_{b}\right) .
$$

2. Let

$$
G_{i}(s)=\left[\begin{array}{c|c}
A_{b}+B_{b} F_{s}-s E_{b} & B_{b} D_{b}^{-1} \\
\hline C_{b}+D_{b} F_{s} & I
\end{array}\right]
$$

and

$$
G_{o}(s)=\left[\begin{array}{c|c}
A-s E & B \\
\hline D_{b} H_{1} & D_{b} H_{2}
\end{array}\right]
$$

where

$$
\left[\begin{array}{ll}
H_{1} & H_{2}
\end{array}\right]:=\left[\begin{array}{llll}
O & -F_{s} & I & O
\end{array}\right] Z^{T} .
$$

Then $G_{i}(s)$ is square inner, the realization (45) is minimal, $G(s)=G_{i}(s) G_{o}(s)$, and $\mathcal{Z}\left(G_{o}\right) \subset \overline{\mathbb{C}}^{-}$.

Proof: 1. From (bi) of Theorem III.2 we have $\Lambda\left(A_{b}-B_{b} D_{b}^{-1} C_{b}-s E_{b}\right) \subset \mathbb{C}^{+}$. Further, from (bii) we conclude that the pair $\left(E_{b}^{-1}\left(A_{b}-B_{b} D_{b}^{-1} C_{b}\right), B_{b} D_{b}^{-1}\right)$ is controllable and standard Lyapunov equation results show that (43) has a unique invertible solution $Y$ which is positive definite $Y>0$. Multiplying (43) to the left and right with $\left(E_{b} Y\right)^{-1}$ and $\left(Y E_{b}^{T}\right)^{-1}$, respectively, we get after some simple manipulations

$$
\begin{gathered}
Y^{-1} E_{b}^{-1}\left(A_{b}+B_{b} F_{s}\right)+\left(A_{b}+B_{b} F_{s}\right)^{T} E_{b}^{-T} Y^{-1} \\
+Y^{-1} E_{b}^{-1} B_{b}\left(D_{b}^{T} D_{b}\right)^{-1} B_{b}^{T} E_{b}^{-T} Y^{-1}=0 .
\end{gathered}
$$

From (bii) of Theorem III.2 we have that the pair $\left(E_{b}^{-1}\left(A_{b}+B_{b} F_{s}\right), Y^{-1} E_{b}^{-1} B_{b} D_{b}^{-1}\right)$ is controllable, and $Y^{-1}>0$. Standard Lyapunov results applied to (48) lead to the conclusion that $\Lambda\left(A_{b}+B_{b} F_{s}-s E_{b}\right) \subset \mathbb{C}^{-}$.

2. This part of the proof is very similar to the proof of part 2 of Theorem IV.1 and thus we only outline the differences. We use (45) to write a realization of

$$
\begin{aligned}
G_{i}^{-1}(s) & =\left[\begin{array}{c|c}
A_{i}-s E_{i} & B_{i} \\
\hline C_{i} & D_{i}
\end{array}\right] \\
& =\left[\begin{array}{c|c}
A_{b}-B_{b} D_{b}^{-1} C_{b}-s E_{b} & B_{b} D_{b}^{-1} \\
\hline-\left(C_{b}+D_{b} F_{s}\right) & I
\end{array}\right] .
\end{aligned}
$$

By direct substitution we check that the equations (28) hold for $X_{s}=E_{b}^{-T} Y^{-1} E_{b}^{-1}$ which shows that $G_{i}(s)^{-1}$ is all-pass (see [50]). Thus $G_{i}(s)$ is inner. Moreover, since $X_{s}$ is invertible we get that (45) is minimal. The rest of the proof follows mutatis mutandis from the proof of Theorem IV.1.

We comment now on alternative existing methods of dislocating zeros and their applicability. Dislocation of zeros by using square inner factors was first performed in [39], [40], but the method is limited to proper rational matrices $G(s)$. The first paper that reports on zero dislocation of an improper $G(s)$ by square inner factors is [41]. In both [40], [41], zeros are dislocated only one-by-one (or in conjugated 
pairs), in a sequential way, and the algorithms are applicable to a non-surjective $G(s)$ as well. However, for those seeking to apply the methods of [40], [41] we caution that dislocating zeros without first dislocating the left minimal indices (i.e., performing on a non-surjective $G(s)$ ) could have perverse effects as instead of dislocating "bad" zeros and replacing them with "good" ones we may increase the sum of left minimal indices. Loosely speaking, if $G(s)$ is not surjective and no special care is taken, we may replace bad zeros with minimal indices which are even worse for the problems at hand. This is now illustrated by an example.

Example IV.4: Let

$$
G(s)=\left[\begin{array}{c}
\frac{s-1}{s+2} \\
\frac{s-1}{s+2}
\end{array}\right]
$$

which has one "bad" zero at $s=1$, one pole at $s=-2$, and $n_{r}=0$ and $n_{\ell}=0$. In fact, it is easy to see that a row minimal basis of the left null space is $\left[\begin{array}{ll}1 & -1\end{array}\right]$. Then

$$
G_{1}(s)=\left[\begin{array}{cc}
\frac{s-1}{s+1} & 0 \\
0 & 1
\end{array}\right]
$$

is inner and let

$$
G_{2}(s):=G_{1}^{-1}(s) G(s)=\left[\begin{array}{c}
\frac{s+1}{s+2} \\
\frac{s-1}{s+2}
\end{array}\right]
$$

which has no "bad" zeros, has one pole at $s=-2$, has no right minimal indices and has a minimal index to the left equal to 1 . It is easy to figure out that $\left[\begin{array}{ll}s-1 & -(s+1)\end{array}\right]$ is a minimal basis for the left null space. Hence form $G(s)=G_{1}(s) G_{2}(s)$ we see that the "bad" zero at 1 was dislocated by left multiplication with a square inner factor, but in a completely unfortunate way as it was replaced with a minimal index to the left.

The method of zero-dislocation described in this paper is closer to [40] rather than [23], [41]. Similarly to [40], our entire reasoning is made on the zeros of the original $G(s)$ and not on the poles of a generalized inverse of $G(s)$. We believe that this brings more insight to the theoretical aspects of the problem.

\section{Solution to the FACTORIZATION PROBlEmS}

In this section we explain briefly how we can apply the already obtained results to compute the inner-outer factorization and the spectral factorization in the most general setting.

\section{A. Solution to the inner-outer factorization problem}

Let $G(s)$ be an arbitrary rational matrix analytic in $\mathbb{C}^{+}$ (i.e., $G(s)$ is weakly stable).

Step 1. Use Theorem IV.1 to determine a factorization

$$
G(s)=G_{i}^{(1)}(s) G_{o}^{(1)}(s)
$$

where

$$
G_{i}^{(1)}(s)=\left[G_{i 1}^{(1)}(s) G_{i 2}^{(1)}(s)\right], G_{o}^{(1)}(s)=\left[\begin{array}{c}
G_{o 1}^{(1)}(s) \\
O
\end{array}\right]
$$

The resulting $G_{i}^{(1)}(s)$ has all poles in $\mathbb{C}^{-}$and it is inner and square, while $G_{o 1}^{(1)}(s)$ is surjective with $n_{b}$ zeros in $\mathbb{C}^{+}$.

Step 2. Use Theorem IV.3 to determine a factorization

$$
G_{o 1}^{(1)}(s)=G_{i}^{(2)}(s) G_{o}^{(2)}(s)
$$

where $G_{i}^{(2)}(s)$ has all poles in $\mathbb{C}^{-}$and it is inner (and square), while $G_{o}^{(2)}(s)$ is surjective with all zeros in $\overline{\mathbb{C}}^{-}$ (i.e., it is outer).

Result. The inner-outer factorization results as

$$
G(s)=G_{i}(s) G_{o}(s)
$$

where

$$
G_{i}(s):=G_{i 1}^{(1)}(s) G_{i}^{(2)}(s), G_{o}(s):=G_{o}^{(2)}(s) .
$$

\section{B. Solution to the spectral factorization problem}

Let $G(s)$ be an arbitrary rational matrix.

Step 0. Use a particular version of Theorem 5.2 in [30] to determine a minimal degree coprime factorization of $G(s)$ in the form

$$
G(s)=M^{-1}(s) N(s)
$$

where $N(s)$ has all its poles in $\overline{\mathbb{C}}^{-}$and $M(s)$ is inner. Steps 1-2. By using the method described above at A., perform an inner-outer factorization of $N(s)$ in the form

$$
N(s)=G_{i}^{N}(s) G_{o}^{N}(s) .
$$

The spectral factorization of $G(s)$ results as

$$
G^{*}(s) G(s)=N^{*}(s) N(s)=G_{o}^{*}(s) G_{o}(s)
$$

where $G_{o}(s):=G_{o}^{N}(s)$ is the spectral factor.

\section{Minimal realizations of the factors}

Throughout the paper we have assumed that the realization of $G(s)$ is weak minimal and not necessarily minimal, i.e., should satisfy conditions (i)-(iv) in (9) only. Weak minimality instead of minimality is important from a numerical viewpoint since if we start with an arbitrary realization for $G(s)$ we can always compute a realization satisfying (i)-(iv) by using orthogonal transformations only. However, a minimal realization satisfying also (v) in (9) could in general be obtained only with the pay-back of using non-orthogonal transformations.

From the proofs of Theorems III.1, III.2, IV.3, and IV.1 it follows that for our method controllability and $\mathbb{C}^{+}$observability are not only sufficient, but also necessary. Indeed, sufficiency follows from the fact that the system pencil associated with such a realization that is not weak minimal has the same left Kronecker structure and the same zeros in $\mathbb{C}^{+}$as the system pencil associated with a weak minimal realization. In other words, point 3 . for $\mathbb{C}^{+}$zeros and point 5 . of Theorem II.1 still hold for a realization which is controllable and $\mathbb{C}^{+}$-observable only. Necessity follows from the fact that non-controllability can lead to an 
increase in the sum of the left Kronecker indices of $\mathcal{S}_{G}(s)$ (and thus 5. of Theorem II.1 fails to hold) while nonobservability can lead to an increase in the number of $\mathbb{C}^{+}$ zeros of $\mathcal{S}_{G}(s)$. If either of these two conditions fails to hold, the whole proposed procedure will collapse.

Nonetheless, if the initial realization is not weak minimal but is controllable and $\mathbb{C}^{+}$observable the whole procedure remains valid but the resulting $G_{o}(s)$ will not be weak minimal.

Even if we start with a weak minimal realization (8) we may obtain, after compressing the rows of $G(s)$, a realization (24) of $G_{o 1}(s)$ that is not weak minimal. As already explained in the proof of Theorem IV.1, weak nonminimality may occur if in the product $G_{o}(s)=G_{i}(s)^{-1} G(s)$ a cancellation between a zero of $G_{i}(s)^{-1}$ and a pole of $G(s)$ takes place (see [18]). However, the resulting realization (24) is controllable and $\mathbb{C}^{+}$observable and thus we may use further this realization for dislocating the zeros without removing first the unobservable part as is apparently required by Theorem IV.3. We conclude by noting that we can remove the possibility of occurrence of (weak) nonminimality in the resulting realization of $G_{o}(s)$ either at the very beginning (see [18]), or at each factorization step, or at the very end. Irrespective of which alternatives we choose, the realizations of the all-pass factors and of the resulting inner factors are always minimal.

\section{Pseudoinverses of RATional matrices}

A straightforward application of the inner-outer factorization is the computation of a generalized (Moore-Penrose type) (pseudo)-inverse $G^{\#}(s)$ of an arbitrary rational matrix $G(s)$. Recall from [28] that $G^{\#}(s)$ is a Moore-Penrose generalized inverse of $G(s)$ if it satisfies the four axioms:

(i) $\quad G(s) G^{\#}(s) G(s)=G(s)$,

(ii) $G^{\#}(s) G(s) G^{\#}(s)=G^{\#}(s)$,

(iii) $G(s) G^{\#}(s)=\left(G(s) G^{\#}(s)\right)^{*}$,

(iv) $G^{\#}(s) G(s)=\left(G^{\#}(s) G(s)\right)^{*}$.

Depending on the interpretation of the conjugation operator $(\cdot)^{*}$, we get different pseudoinverses for the same rational matrix $G(s)$. We illustrate for the case in which the operator is conjugation in continuous-time.

To compute the generalized inverse, we perform successively a row and a column compression of $G(s)$ as follows:

$$
G(s)=U(s)\left[\begin{array}{c}
G_{1}(s) \\
O
\end{array}\right]
$$

where $G_{1}(s)$ is surjective and $U(s)$ is square inner, and

$$
G_{1}^{T}(s)=V^{T}(s)\left[\begin{array}{c}
G_{2}^{T}(s) \\
O
\end{array}\right]
$$

where $G_{2}(s)$ is invertible and $V^{T}(s)$ is square inner. We get the overall decomposition

$$
G(s)=U(s)\left[\begin{array}{cc}
G_{2}(s) & O \\
O & O
\end{array}\right] V(s) .
$$

Define

$$
G^{\#}(s):=V^{*}(s)\left[\begin{array}{cc}
G_{2}^{-1}(s) & O \\
O & O
\end{array}\right] U^{*}(s) .
$$

Then $G^{\#}(s)$ fulfills all four axioms (i)-(iv) above, and thus, it is the unique Moore-Penrose generalized inverse of $G(s)$ (with respect to the conjugation operator in continuoustime). Notice that the rational decomposition (51) of $G(s)$ generalizes the complete orthogonal decomposition of a real matrix.

\section{NUMERICAL ASPECTS}

In this section we comment on the numerical algorithms that are recommended at each step of the factorization process and further numerical refinements.

\section{Computation of Kronecker-like forms}

These are needed at Step 4 of the spectral decomposition in both Theorems III.1 and III.2, and any of the existing staircase algorithms can be used as basic ingredient [37], [5], [42], [29]. While the algorithm in [37] is perhaps the most reliable as its rank decisions are based on singular value decompositions, the pay-back is its complexity $\mathcal{O}\left(n^{4}\right)$ due to the inherent recursive nature of the staircase decomposition. Alternative $\mathcal{O}\left(n^{3}\right)$ algorithms are given in [5], [29] where the rank decisions are based on orthogonal QR-decompositions with column pivoting.

To obtain the Kronecker-like form needed at Step 4 in Theorem III.1 we have to use a staircase algorithm twice to split the left singular Kronecker structure from the right singular Kronecker structure and the generalized eigenvalues (regular part) (see for example [5], [29]).

\section{Riccati and Lyapunov equations}

The equation (21) is a standard continuous-time algebraic Riccati equation and has always a stabilizing solution. For computing its stabilizing solution one has to solve a standard Hamiltonian eigenvalue problem [3] or can solve a generalized Hamiltonian eigenvalue problem with the benefit of avoiding the inversion of $D_{\ell}$ both for computing the solution and the stabilizing feedback (see [20]). The price to pay is that a generalized eigenvalue problem of dimension $2 n_{\ell}+r$ instead of $2 n_{\ell}$ has to be solved. For computing the unique positive definite solution to the generalized Lyapunov equation with positive free term (43) one can use the algorithms in [17], [32].

Global algorithmic aspects

We can see from the proofs of Theorems III.1, III.2, that we can construct two orthogonal matrices $Q$ and $Z$ such that

$$
\left[\begin{array}{cc}
A-s E & B \\
\hdashline Q^{T} C & Q^{T} D
\end{array}\right] Z=
$$




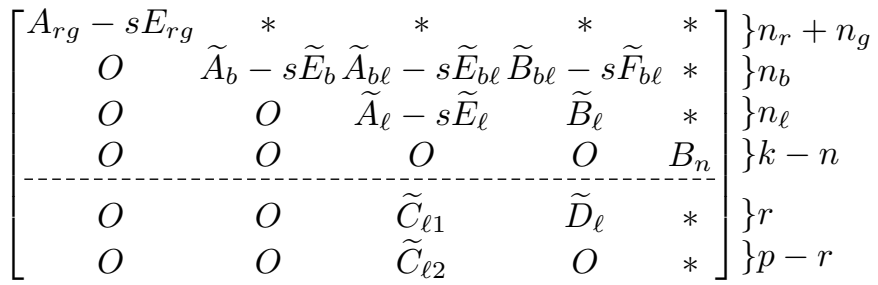

where $A_{r g}-s E_{r g}$ contains the right Kronecker structure and the generalized eigenvalues in $\overline{\mathbb{C}}^{-}, \widetilde{A}_{b}-s \widetilde{E}_{b}$ contains the generalized eigenvalues in $\mathbb{C}^{+}$, and the subpencil

$$
\left[\begin{array}{cc}
\widetilde{A}_{\ell}-s \widetilde{E}_{\ell} & \widetilde{B}_{\ell} \\
\widetilde{C}_{\ell 1} & \widetilde{D}_{\ell} \\
\widetilde{C}_{\ell 2} & O
\end{array}\right]
$$

contains the left Kronecker structure. We may proceed further in two alternative ways: either solving a Riccati equation of dimension $n_{\ell}$, performing a reordering of generalized eigenvalues, and finally solving a Lyapunov equation of order $n_{b}$, or solving instead a single Riccati equation of order $n_{\ell}+n_{b}$. We give now details of both these alternatives.

Solving a Riccati equation of dimension $n_{\ell}$. Once the decomposition (53) is computed, we apply Theorem IV.1 to compress the rows of $G(s)$ and get $G_{i}(s)$ and $G_{o}(s)$ given in (23) and (24), respectively. Then a simple update provides the decomposition (15) needed in Theorem IV.3 while completely avoids Steps 1-4 in Theorem III.2. Indeed, Theorem IV.3 applies to the resulting $G_{01}(s)$ in (24) whose system pencil is in the form (see (37) and (53))

$$
\mathcal{S}_{G_{o 1}}(s) Z=\left[\begin{array}{ccccc}
A_{r g}-s E_{r g} & * & * & * & * \\
O & \widetilde{A}_{b}-s \widetilde{E}_{b} & * & * & * \\
O & O & \widetilde{A}_{\ell}-s \widetilde{E}_{\ell} \widetilde{B}_{\ell} & * \\
O & O & O & O & \widetilde{B}_{n} \\
\hdashline O & O & -\widetilde{D}_{\ell} F_{s} & \widetilde{D}_{\ell} & *
\end{array}\right] .
$$

We use (54) as the starting point to get the decomposition in Theorem III.2 as follows. We determine an orthogonal $Z_{0}$ such that $\left[\begin{array}{ll}-\widetilde{D}_{\ell} F_{s} & \widetilde{D}_{\ell}\end{array}\right] Z_{0}=\left[\begin{array}{ll}O & \bar{D}_{l}\end{array}\right]$ with $\bar{D}_{\ell}$ invertible which is possible since $\widetilde{D}_{\ell}$ is invertible. We get

$$
\begin{gathered}
\mathcal{S}_{G_{o 1}}(s) Z \operatorname{diag}\left(I, Z_{0}\right) \\
=\left[\begin{array}{ccccc}
A_{r g}-s E_{r g} & * & * & * & * \\
O & A_{b}-s E_{b} & * & * & * \\
O & O & \widehat{A}_{\ell}-s \widehat{E}_{\ell} \widehat{B}_{\ell}-s \widehat{F}_{\ell} & * \\
O & O & O & O & B_{n} \\
\hdashline O & O & O & \bar{D}_{\ell} & *
\end{array}\right]
\end{gathered}
$$

and because $\Lambda\left(\widetilde{A}_{\ell}+\widetilde{B}_{\ell} F_{s}-s \widetilde{E}_{\ell}\right) \subset \mathbb{C}^{-}$we get $\Lambda\left(\widetilde{A}_{\ell}+\right.$ $\left.\widetilde{B}_{\ell} F_{s}-s \widetilde{E}_{\ell}\right)=\Lambda\left(\widehat{A}_{\ell}-s \widehat{E}_{\ell}\right) \subset \mathbb{C}^{-}$. Now we determine two orthogonal transformations such that

$$
U_{0}\left[\begin{array}{cc}
\widetilde{A}_{b}-s \widetilde{E}_{b} & * \\
O & \widehat{A}_{\ell}-s \widehat{E}_{\ell}
\end{array}\right] V_{0}=\left[\begin{array}{cc}
\bar{A}_{\ell}-s \bar{E}_{\ell} & * \\
O & \bar{A}_{b}-s \bar{E}_{b}
\end{array}\right]
$$

where $\Lambda\left(\widetilde{A}_{b}-s \widetilde{E}_{b}\right)=\Lambda\left(\bar{A}_{b}-s \bar{E}_{b}\right) \subset \mathbb{C}^{+}$and $\Lambda\left(\widehat{A}_{\ell}-s \widehat{E}_{\ell}\right)=$ $\Lambda\left(\bar{A}_{\ell}-s \bar{E}_{\ell}\right) \subset \mathbb{C}^{-}$. Matrices $U_{0}$ and $V_{0}$ can be determined by using a $Q Z$ algorithm [27] followed by a reordering of the eigenvalues [38]. With adequate updating we are now in the position of Step 5 of the proof of Theorem III.2 and we may further proceed analogously. For swapping generalized eigenvalues one can use the algorithm in [38]. It is always possible to take advantage of the fact that after swapping the eigenvalues in (56) (and before applying Step 5) the resulting pencil $\bar{A}_{b}-s \bar{E}_{b}$ is already in a quasi-triangular generalized real Schur form. Then Step 5 in the proof of Theorem III.2 can be skipped and the Lyapunov equation to be solved becomes (see (19))

$$
\begin{gathered}
\bar{A}_{b} Y \bar{E}_{b}^{T}+\bar{E}_{b} Y \bar{A}_{b}^{T} \\
-\left(\bar{B}_{b}-\bar{A}_{b} \bar{E}_{b}^{-1} \bar{F}_{b}\right)\left(\bar{D}_{b}^{T} \bar{D}_{b}\right)^{-1}\left(\bar{B}_{b}-\bar{A}_{b} \bar{E}_{b}^{-1} \bar{F}_{b}\right)^{T}=0 .
\end{gathered}
$$

The disadvantage of solving (57) instead of (43) is that the inverse of $E_{b}$ appears unavoidably in its coefficients.

Solving a Riccati equation of dimension $n_{\ell}+n_{b}$. We start again from (53). Since $\widetilde{E}_{b}$ is invertible, there exists an orthogonal $Z_{0}$ such that

$$
\left[\begin{array}{ll}
\widetilde{E}_{b} & \widetilde{F}_{b \ell}
\end{array}\right] Z_{0}=\left[\begin{array}{cc}
\widehat{E}_{b} & O
\end{array}\right]
$$

where $\widehat{E}_{b}$ is invertible. With an appropriate updating of $Z$ in (53) we get

$$
\left[\begin{array}{cc}
A-s E & B \\
\hdashline Q^{T} C & Q^{T} D
\end{array}\right] Z
$$

$$
=\left[\begin{array}{ccccc}
A_{r g}-s E_{r g} & * & * & * & * \\
O & \widehat{A}_{b}-s \widehat{E}_{b} & \widetilde{A}_{b \ell}-s \widetilde{E}_{b \ell} & \widehat{B}_{b \ell} & * \\
O & \widehat{A}_{\ell b} & \widetilde{A}_{\ell}-s \widetilde{E}_{\ell} & \widehat{B}_{\ell} & * \\
O & O & O & O & \widetilde{B}_{n} \\
\hdashline O & \widehat{C}_{\ell 1} & \widetilde{C}_{\ell 1} & \widehat{D}_{\ell} & * \\
O & O & \widetilde{C}_{\ell 2} & O & *
\end{array}\right] \begin{aligned}
& \} n_{r}+n_{g} \\
& \} n_{\ell} \\
& \} r-n \\
& \} p-r
\end{aligned}
$$

Now we put

$$
\begin{array}{ccc}
A_{\ell}-s E_{\ell}:=\left[\begin{array}{ccc}
\widehat{A}_{b}-s \widehat{E}_{b} & \widetilde{A}_{b \ell}-s \widetilde{E}_{b \ell} \\
\widehat{A}_{\ell b} & \widetilde{A}_{\ell}-s \widetilde{E}_{\ell}
\end{array}\right], B_{\ell}:=\left[\begin{array}{c}
\widehat{B}_{b \ell} \\
\widehat{B}_{\ell}
\end{array}\right] \\
C_{\ell 1}:=\left[\begin{array}{ll}
\widehat{C}_{\ell 1} & \widetilde{C}_{\ell 1}
\end{array}\right], & D_{\ell}:=\widehat{D}_{\ell} \\
C_{\ell 2}:=\left[\begin{array}{ll}
O & \widetilde{C}_{\ell 2}
\end{array}\right] &
\end{array}
$$

and get finally a decomposition of the form

$$
\begin{aligned}
& {\left[\begin{array}{cc}
I & O \\
O & Q^{T}
\end{array}\right]\left[\begin{array}{cc}
A-s E & B \\
\hdashline & D
\end{array}\right] Z} \\
& {\left[\begin{array}{cccc}
A_{r g}-s E_{r g} & * & * & * \\
O & A_{\ell}-s E_{\ell} & B_{\ell} & * \\
O & O & O & B_{n} \\
\hdashline O & C_{\ell 1} & D_{\ell} & * \\
O & C_{\ell 2} & O & *
\end{array}\right] \begin{array}{l}
\} n_{r}+n_{g} \\
\} n_{\ell}+n_{b} \\
\} k-n \\
\} r \\
\} p-r
\end{array}} \\
& \underbrace{}_{\begin{array}{c}
n_{r}+n_{g}+ \\
+m-r
\end{array}} \overbrace{n_{\ell}+n_{b}} \overbrace{r-n}
\end{aligned}
$$


where

(I) The pencil $A_{r g}-s E_{r g}$ is surjective and its zeros are given by $\mathcal{Z}(G(s)) \cap \overline{\mathbb{C}}^{-}$;

(II) $E_{\ell}, D_{\ell}, B_{n}$ are invertible, the pencil

$$
\left[\begin{array}{cc}
A_{\ell}-s E_{\ell} & B_{\ell} \\
C_{\ell 1} & D_{\ell} \\
C_{\ell 2} & O
\end{array}\right]
$$

is injective and its zeros are given by $\widetilde{A}_{b}-s \widetilde{E}_{b}=$ $\mathcal{Z}(G(s)) \cap \mathbb{C}^{+}$.

We get the following theorem which merges into a whole Theorems IV.1 and IV.3.

Theorem VII.1: Let $G(s)$ be a real rational matrix with a weak minimal realization (8), and let $Q$ and $Z$ be orthogonal matrices as in (59) such that conditions (I) and (II) above hold. Then we have:

1. The continuous-time algebraic Riccati equation (21) has a positive semidefinite stabilizing symmetric solution $X_{s}$ such that $\Lambda\left(A_{\ell}+B_{\ell} F_{s}-s E_{\ell}\right) \subset \mathbb{C}^{-}$, where $F_{s}$ is given by $(22)$ and $C_{\ell}^{T}:=\left[\begin{array}{cc}C_{\ell 1}^{T} & C_{\ell 2}^{T}\end{array}\right]$.

2. The rational matrix $G_{i}(s)$ given by $(23)$ is square inner, the realization (23) is minimal, $G(s)=G_{i}(s) G_{o}(s)$, where $G_{o}(s)$ is given by $(24)$, and $G_{o 1}(s)$ is surjective and has no zeros in $\mathbb{C}^{+}$(i.e., it is outer).

From the above theorem we see that instead of solving a Riccati equation (21) of order $n_{\ell}$, swapping generalized eigenvalues as in (56), and solving a Lyapunov equation (43) (or (57)) of order $n_{b}$, we can alternatively solve one single Riccati equation (21) of order $n_{b}+n_{\ell}$. Further, we can prove that this Riccati equation of order $n_{b}+n_{\ell}$ actually decouples into a Riccati equation of order $n_{\ell}$, a Lyapunov equation of order $n_{b}$ and a Sylvester equation of order $n_{b} \times n_{\ell}$. Overall, this comes down to solving a Sylvester equation instead of swapping generalized eigenvalues. This could bring numerical benefits in terms of number of operations as instead of swapping by orthogonal transformations two square blocks of dimensions $n_{b}$ and $n_{\ell}$, one solves a Sylvester equation of dimension $n_{b} \times n_{\ell}$. Nonetheless, this could result as well in a loss of accuracy since non-orthogonal transformations are involved instead of orthogonal ones. In fact, for both these alternatives the numerical accuracy is dependent on the separation of the spectra of $\widetilde{A}_{b}-s \widetilde{E}_{b}$ and $\widetilde{A}_{\ell}+\widetilde{B}_{\ell} F_{s}-s \widetilde{E}_{\ell}$, in (53), where $F_{s}$ is the stabilizing Riccati feedback of the Riccati equation of dimension $n_{\ell}$.

\section{Recursive dislocation}

The dislocation of zeros can alternatively be obtained in a recursive fashion, and the corresponding inner factor will result as a product of first or second degree elementary inner factors [40], [41]. Regardless of whether we employ an "one-shot" Lyapunov equation based approach or a sequential procedure for obtaining the zeros dislocation, we need the pencil $A_{b}-B_{b} D_{b}^{-1} C_{b}-s E_{b}$ in (43) (or $\bar{A}_{b}-s \bar{E}_{b}$ in $(57))$ to be in a generalized real Schur form. This implies solving in fact a generalized eigenvalue problem of dimension $n_{b}$ that computes the "values" of the zeros that will be dislocated. The recursive dislocation of zeros involves basically the solution of generalized Lyapunov equations of order 1 or 2, local updates of the Kronecker-like form followed by a reordering of the generalized eigenvalues. In comparison with the "one-shot" approach, the sequential approach needs in addition a reordering of the eigenvalues at each step, and this results in an overall increase in the number of operations. In turn, the inner factor results as a product of elementary inner factors with an arbitrarily chosen order and this may be useful in certain applications

The dislocation of minimal indices (row compression) can be obtained in a recursive way as well, although the implementation details are more hairy. In this case we have to solve first a Hamiltonian eigenvalue problem of dimension $2 n_{\ell}$ to determine the "values" of the zeros in which will be dislocated the minimal indices, and this can be done more efficiently than solving the Riccati equation by the algorithms in [6]. Again, we can obtain an inner factor in an already factorized form.

In conclusion, the recursive approach is recommended only if a particular factorized form of the inner factor or the generalized Schur form of the system pencil is needed at further steps.

\section{NUMERICAL EXAMPLES}

In this section we illustrate the proposed approach by several numerical examples. For the computation of the inner-outer factorization we have used the DESCRIPTOR Systems Toolbox [43] implemented under Matlab 5.1. This Toolbox uses extensively the object oriented approach for control systems analysis and design introduced within the Control Toolbox 4.0. For all critical computations, the .mex files technology of MATLAB has been used, on basis of FORTRAN codes from LAPACK [2] and SLICOT [7] libraries.

The rational expressions of the computed factors have been converted to exact expressions using symbolic manipulations with Maple V Release 5. For tutorial purposes we have performed the computations step-by-step and we have used at several steps non-orthogonal transformations as well.

Example 1. Consider the proper rational matrix

$$
G(s)=\left[\begin{array}{ccc}
\frac{s-1}{(s+2)} & \frac{s}{(s+2)} & \frac{1}{(s+2)} \\
0 & \frac{s-2}{(s+1)^{2}} & \frac{s-2}{(s+1)^{2}} \\
\frac{s-1}{s+2} & \frac{s^{2}+2 s-2}{(s+1)(s+2)} & \frac{2 s-1}{(s+1)(s+2)}
\end{array}\right] .
$$

The structural elements of $G(s)$ are: zeros at 1,2 , and $\infty$, all of order 1 ; poles at $-1,-2$ both of order 2 ; normal rank $r=2$; one right minimal index equal to 0 ; one left minimal index equal to 1 . Thus $n_{z}=3, n_{b}=2, n_{g}=1, n_{\ell}=1$, $n_{r}=0$. Therefore, none of known methods is applicable to compute the inner-outer factorization of $G(s)$.

1. We compute first an appropriate realization as required by Theorem III.1 and the corresponding decomposition (11). A minimal standard state space realization for 
$G(s)$ is given by

$$
\begin{array}{ll}
A=\left[\begin{array}{rrrr}
-4 & 1 & 0 & 0 \\
-9 & 2 & 0 & 0 \\
2 & -1 & -2 & 0 \\
-1 & 0 & 0 & -2
\end{array}\right], \quad B=\left[\begin{array}{ccc}
0 & 1 & 1 \\
0 & 0 & 0 \\
-3 & -3 & 0 \\
0 & 0 & 0
\end{array}\right], \\
C=\left[\begin{array}{llll}
1 & 0 & 1 & 0 \\
1 & 0 & 0 & 0 \\
2 & 0 & 1 & 1
\end{array}\right], & D=\left[\begin{array}{lll}
1 & 1 & 0 \\
0 & 0 & 0 \\
1 & 1 & 0
\end{array}\right] .
\end{array}
$$

We chose this minimal realization such that with $U=V$ $=I$

$$
\begin{gathered}
Q=\left[\begin{array}{l:l}
Q_{1} & Q_{2}
\end{array}\right]=\left[\begin{array}{rrrrr}
-\sqrt{2} / 2 & \sqrt{6} / 6 & -\sqrt{3} / 3 \\
0 & -\sqrt{6} / 3 & -\sqrt{3} / 3 \\
-\sqrt{2} / 2 & -\sqrt{6} / 6 & \sqrt{3} / 3
\end{array}\right], \\
Z=\left[\begin{array}{rrrrrrr}
0 & 0 & 0 & 0 & 0 & 0 & -1 \\
0 & 0 & 1 & 0 & 0 & 0 & 0 \\
0 & 0 & 0 & 1 & 0 & 0 & 0 \\
0 & 0 & 0 & 0 & 1 & 0 & 0 \\
-1 & 0 & 0 & -1 & -1 & -1 & 2 \\
1 & 0 & 0 & 0 & 0 & 0 & 0 \\
-1 & 1 & -1 & 0 & 0 & 0 & 0
\end{array}\right],
\end{gathered}
$$

we get

$$
\begin{aligned}
& \operatorname{diag}\left(I_{4}, Q^{T}\right)\left[\begin{array}{cc}
A-s I & B \\
============ \\
C & D
\end{array}\right] Z
\end{aligned}
$$

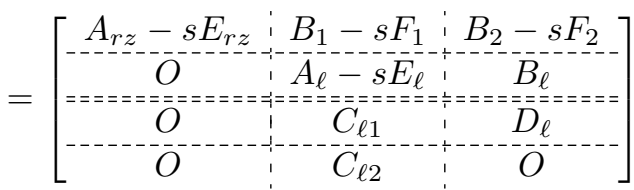

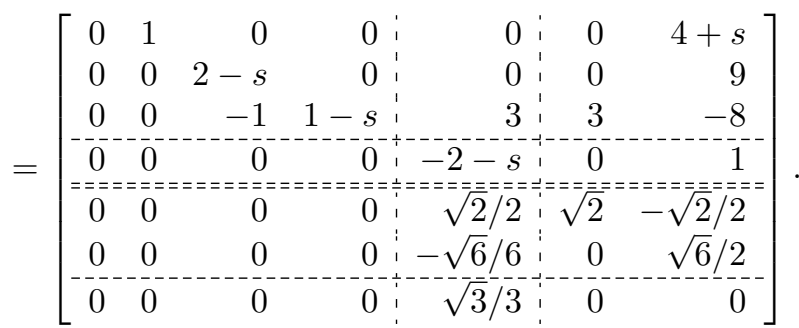

2. We solve now the Riccati equation (21) for $X_{s}$ and compute the corresponding stabilizing feedback $F_{s}$. We get

$$
X_{s}=(3 \sqrt{3}-5) / 2, \quad F_{s}=\left[\begin{array}{c}
(1-\sqrt{3}) / 2 \\
2-\sqrt{3}
\end{array}\right]
$$

The partial inner factor $G_{i}^{(1)}(s)$ and $G_{o}^{(1)}(s)=\left(G_{i}^{(1)}\right)^{-1}(s) G(s)$ are computed as follows

$$
\begin{aligned}
G_{i}^{(1)}(s) & =Q\left[\begin{array}{ccc}
A_{\ell}+B_{\ell} F_{s}-s I & B_{\ell} D_{\ell}^{-1} & O \\
\hline C_{\ell 1}+D_{\ell} F_{s} & I & O \\
C_{\ell 2} & O & I
\end{array}\right] \\
= & {\left[\begin{array}{ccc}
-\frac{\sqrt{2}}{2} & \frac{\sqrt{6}(s+1)}{6(s+\sqrt{3})} & -\frac{\sqrt{3}(s-2)}{3(s+\sqrt{3})} \\
0 & -\frac{\sqrt{6}(s+2)}{3(s+\sqrt{3})} & -\frac{\sqrt{3}(s-1)}{3(s+\sqrt{3})} \\
-\frac{\sqrt{2}}{2} & -\frac{\sqrt{6}(s+1)}{6(s+\sqrt{3})} & \frac{\sqrt{3}(s-2)}{3(s+\sqrt{3})}
\end{array}\right] }
\end{aligned}
$$

$$
\begin{aligned}
& G_{o}^{(1)}(s)=\left[\begin{array}{c|c}
G_{o 1}^{(1)}(s) \\
\hdashline O
\end{array}\right]=\left[\begin{array}{c|c}
A-s I & B \\
\hline D_{\ell} H_{1} & D_{\ell} H_{2} \\
\hdashline O & O
\end{array}\right] \\
& =\left[\begin{array}{ccc}
-\frac{\sqrt{2}(s-1)}{s+2} & -\frac{\sqrt{2}(2 s-1)}{2(s+1)} & -\frac{3 \sqrt{2} s}{2(s+1)(s+2)} \\
0 & -\frac{\sqrt{6}(s-2)(s+\sqrt{3})}{2(s+1)^{2}(s+2)} & -\frac{\sqrt{6}(s-2)(s+\sqrt{3})}{2(s+1)^{2}(s+2)} \\
0 & 0 & 0
\end{array}\right],
\end{aligned}
$$

where

$$
\begin{gathered}
{\left[\begin{array}{lll}
O & -F_{s} & I
\end{array}\right] Z^{-1}:=\left[\begin{array}{ll:lll}
H_{1} & H_{2}
\end{array}\right]} \\
=\left[\begin{array}{rrrr:rrr}
0 & 1 & 4 & -1 & 0 & 0 & 0 \\
0 & 0 & -2 & -1 & 1 & 0 & 1
\end{array}\right] .
\end{gathered}
$$

3. To dislocate the zeros in $\mathbb{C}^{+}$of $G_{o 1}^{(1)}(s)$ we determine $\widehat{U}$ and $\widehat{Z}$

$$
\widehat{U}=\left[\begin{array}{rrrrrr}
1 & 0 & 0 & 0 \\
0 & 0.5385 & 0.1235 & -0.8335 \\
0 & 0.5744 & -0.7776 & 0.2559 \\
0 & 0.6165 & 0.6165 & 0.4896
\end{array}\right], \quad \text { }
$$

such that

$$
\begin{aligned}
& \operatorname{diag}\left(\widehat{U}, I_{2}\right)\left[\begin{array}{cc}
A-s I & B \\
=================== \\
D_{\ell} H_{1} & D_{\ell} H_{2}
\end{array}\right] \widehat{Z} \\
& =\left[\begin{array}{c:c:c}
A_{r g}-s E_{r g} & B_{1}-s F_{1} & B_{2}-s F_{2} \\
\hdashline O & A_{b}-s E_{b} & B_{b}-s F_{b} \\
\hdashline============-======= & D & D_{b}
\end{array}\right] \\
& =\left[\begin{array}{ccc}
0 & 1 & -0.8932-0.2233 s \\
0 & 0 & -1.7321-s
\end{array}\right.
\end{aligned}
$$

$$
\left.\begin{array}{cc:cr}
0.2742+0.0686 s & 0.5247+0.1312 s & 0 & 4+s \\
1.1458 & 1.9921 & 0.3704 & 3.0254 \\
\hdashline 2-s & 1.2612 & -2.3327 & 11.6457 \\
0 & 1-s & 1.8496 & 1.1062 \\
\hdashline============================ & 0============ \\
0 & 0 & 1.4142 & -0.7071 \\
0 & 0 & 1.2247
\end{array}\right] .
$$

The solution to the Lyapunov equation (43) and the corresponding feedback matrix (22) are

$$
\widehat{Y}=\left[\begin{array}{rr}
17.0464 & 3.0733 \\
3.0733 & 2.2304
\end{array}\right], \widehat{F}_{s}=\left[\begin{array}{ll}
-0.0093 & -0.7053 \\
-0.3997 & -0.0564
\end{array}\right] \text {. }
$$


The inner factor $G_{i}^{(2)}(s)$ which dislocates the zeros in $\mathbb{C}^{+}$ is

$$
\begin{aligned}
G_{i}^{(2)}(s) & =\left[\begin{array}{c|cc}
A_{b}+B_{b} \widehat{F}_{s}-s I & B_{b} D_{b}^{-1} & O \\
\hline D_{b} \widehat{F}_{s} & I & O \\
O & O & I
\end{array}\right] \\
& =\left[\begin{array}{ccc}
\frac{s-\alpha}{s+1} & \frac{\beta}{s+1} & 0 \\
\frac{\beta(s-2)}{(s+1)(s+2)} & \frac{(s-2)(s+\alpha)}{(s+1)(s+2)} & 0 \\
0 & 0 & 1
\end{array}\right],
\end{aligned}
$$

where $\alpha=\frac{4+3 \sqrt{3}}{8+3 \sqrt{3}}=0.6969$ and $\beta=2 \frac{\sqrt{3}+3}{8+3 \sqrt{3}}=0.7172$. The factor $G_{o}^{(2)}(s):=\left(G_{i}^{(2)}(s)\right)^{-1} G_{o}^{(1)}(s)$ results as

$$
G_{o}^{(2)}(s)=\left[\begin{array}{c|c}
A-s I & B \\
\hline D_{b} \widehat{H}_{1} & D_{b} \widehat{H}_{2} \\
O & O
\end{array}\right]=
$$

$$
\left[\begin{array}{ccc}
\frac{-1.414 s-0.9855}{s+2} \frac{-1.414 s^{2}-3.107 s-2.014}{(s+1)^{2}} & \frac{-2.121 s^{2}-4.843 s-3.043}{(s+2)(s+1)^{2}} \\
\frac{1.014}{(s+2} & \frac{-0.2104 s-0.9712}{(s+1)^{2}} & \frac{-1.225 s^{2}-3.421 s-2.957}{(s+2)(s+1)^{2}} \\
0 & 0 & 0
\end{array}\right]
$$

where

$$
\begin{gathered}
{\left[\begin{array}{lll}
O & -\widehat{F}_{s} & I
\end{array}\right] \widehat{Z}^{-1}:=\left[\begin{array}{r:rrrr}
\widehat{H}_{1} & \widehat{H}_{2}
\end{array}\right]} \\
=\left[\begin{array}{rrrrrrr}
-2 & 0.4050 & 0.6247 & -0.2216 & -1 & -1 & 0 \\
-1 & 0.2233 & 0.3311 & -0.0748 & 0 & 0 & 0
\end{array}\right]
\end{gathered}
$$

Note that the resulting state space realization of $G_{o}^{(2)}(s)$ is not minimal, having an unobservable pole in -1 . This means that $G(s)$ contains a free inner factor which is now included in $G_{i}(s)$.

For reference purposes we give also the exact expressions of the factors $G_{i}(s):=G_{i}^{(1)}(s) G_{i}^{(2)}(s)=\left[G_{i 1}(s) \mid G_{i 1}(s)\right]$ and $G_{o}(s):=G_{o}^{(2)}(s)$ :

$$
G_{i 1}(s)=\frac{\sqrt{2}}{37}\left[\begin{array}{c}
-\frac{37 s^{3}+(71+15 \sqrt{3}) s^{2}+(-48+55 \sqrt{3}) s+10 \sqrt{3}-76}{2(s+\sqrt{3})(s+1)(s+2)} \\
-\frac{2(-1+5 \sqrt{3})(s-2)}{(s+\sqrt{3})(s+1)} \\
-\frac{37 s^{3}+(67+35 \sqrt{3}) s^{2}+(-44+35 \sqrt{3}) s-30 \sqrt{3}-68}{2(s+\sqrt{3})(s+1)(s+2)}
\end{array}\right.
$$$$
\frac{\sqrt{3}\left(37 s^{3}+(-26-18 \sqrt{3}) s^{2}+(-157-66 \sqrt{3}) s-190-12 \sqrt{3}\right)}{6(s+\sqrt{3})(s+1)(s+2)}
$$$$
-\frac{\sqrt{3}(37 s+5+12 \sqrt{3})(s-2)}{3(s+\sqrt{3})(s+1)}
$$$$
\left.-\frac{\sqrt{3}\left(37 s^{3}+(-38+42 \sqrt{3}) s^{2}+(42 \sqrt{3}-1) s+170-36 \sqrt{3}\right)}{6(s+\sqrt{3})(s+1)(s+2)}\right]
$$

$$
G_{i 2}(s)=\left[\begin{array}{c}
-\frac{\sqrt{3}(s-2)}{3(s+\sqrt{3})} \\
-\frac{\sqrt{3}(s-1)}{3(s+\sqrt{3})} \\
\frac{\sqrt{3}(s-2)}{3(s+\sqrt{3})}
\end{array}\right]
$$

$$
\begin{aligned}
G_{o}(s)=\frac{\sqrt{2}}{37} & {\left[\begin{array}{cc}
-\frac{37 s+5+12 \sqrt{3}}{s+2} & -\frac{74 s^{2}+24 \sqrt{3} s+121 s+95+6 \sqrt{3}}{2(s+1)^{2}} \\
-\frac{2(\sqrt{3}-15)}{s+2} & -\frac{(41 \sqrt{3}-60)(13 s+34+15 \sqrt{3})}{26(s+1)^{2}} \\
0 & 0 \\
& -\frac{3\left(37 s^{2}+81 s+2 \sqrt{3} s+60-4 \sqrt{3}\right)}{2(s+1)^{2}(s+2)} \\
- & \frac{\sqrt{3}\left(37 s^{2}-5 \sqrt{3} s+112 s+10 \sqrt{3}+72\right)}{2(s+1)^{2}(s+2)} \\
0
\end{array}\right] \cdot }
\end{aligned}
$$

Example 2. Using a compression to the left and a compression to the right, both by using all-pass factors, we obtain the unique pseudoinverse of $G(s)$ as

$$
\begin{gathered}
G^{\#}(s)=\frac{1}{9}\left[\begin{array}{cc}
\frac{s^{5}+8 s^{4}+4 s^{3}-28 s^{2}-5 s+38}{s^{4}-3 s^{3}-s^{2}+9 s-6} & \frac{-2 s^{4}-12 s^{3}-20 s^{2}-6 s+4}{s^{3}-s^{2}-3 s+3} \\
\frac{-s^{4}-s^{3}+3 s^{2}-2 s-8}{s^{3}-s^{2}-3 s+3} & \frac{2 s^{4}+3 s^{3}-7 s^{2}-12 s-4}{s^{3}-s^{2}-3 s+3} \\
\frac{-2 s^{5}-7 s^{4}+s^{3}+20 s^{2}+s-22}{s^{4}-3 s^{3}-s^{2}+9 s-6} & \frac{4 s^{4}+15 s^{3}+13 s^{2}-6 s-8}{s^{3}-s^{2}-3 s+3} \\
\frac{-s^{5}-2 s^{4}-4 s^{3}-14 s^{2}+5 s+34}{s^{4}-3 s^{3}-s^{2}+9 s-6} \\
\frac{s^{4}+4 s^{3}+3 s^{2}-7 s-10}{s^{3}-s^{2}-3 s+3} \\
\frac{2 s^{5}+4 s^{4}-s^{3}+s^{2}-s-14}{s^{4}-3 s^{3}-s^{2}+9 s-6}
\end{array}\right] .
\end{gathered}
$$

Example 3. We show how to compute the inner-outer and spectral factorizations for a singular polynomial matrix

$$
G(s)=\left[\begin{array}{ccc}
s^{2}+s+1 & 4 s^{2}+3 s+2 & 2 s^{2}-2 \\
s & 4 s-1 & 2 s-2 \\
s^{2} & 4 s^{2}-s & 2 s^{2}-2 s
\end{array}\right] .
$$

The structural elements of $G(s)$ are: a zero at 1 of order 1; a pole at $\infty$ of order 2 ; normal rank $r=2$; one left minimal index equal to 1 ; one right minimal index equal to 0 . Thus $n_{z}=1, n_{b}=1, n_{g}=0, n_{\ell}=1, n_{r}=0$.

1. We compute first the preliminary decomposition (11). A weak minimal descriptor realization for $G(s)$ is given by

$$
\begin{aligned}
& A-s E=\left[\begin{array}{cccc}
-s & 0 & 1 & 0 \\
0 & -s & 0 & 1 \\
1 & 0 & 0 & 0 \\
0 & 1 & -1 & 0
\end{array}\right], B=\left[\begin{array}{rrr}
0 & 0 & 0 \\
0 & 0 & 0 \\
1 & 4 & 2 \\
0 & -1 & -2
\end{array}\right], \\
& C=\left[\begin{array}{rrrr}
0 & 0 & -1 & -1 \\
0 & 0 & -1 & 0 \\
0 & 0 & 0 & -1
\end{array}\right], \quad D=\left[\begin{array}{rrr}
1 & 2 & -2 \\
0 & -1 & -2 \\
0 & 0 & 0
\end{array}\right] \text {. }
\end{aligned}
$$

With

$$
Q=\left[\begin{array}{rll}
-1 & 0 & 0 \\
0 & 0 & 1 \\
0 & 1 & 0
\end{array}\right], \quad Z=\left[\begin{array}{rrrrrrr}
0 & 1 & 0 & 0 & 0 & 0 & 0 \\
0 & 0 & 1 & 0 & 0 & 0 & 1 \\
0 & 1 & 2 & -1 & -1 & -1 & 0 \\
0 & 0 & 0 & 0 & -1 & 0 & 0 \\
6 & 3 & 4 & -4 & -4 & -3 & 0 \\
-2 & -1 & -1 & 1 & 1 & 1 & 0 \\
1 & 0 & 0 & 0 & 0 & 0 & 0
\end{array}\right]
$$

we get

$$
\operatorname{diag}\left(I_{4}, Q^{T}\right)\left[\begin{array}{cc}
A-s E & B \\
===== \pm== \pm==== \\
C & D
\end{array}\right] Z
$$




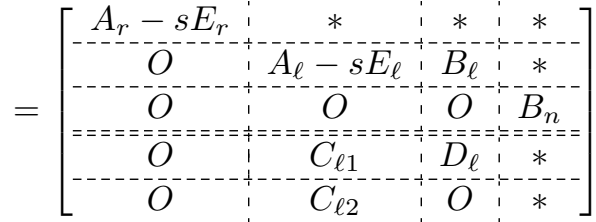

$$
\begin{aligned}
& =\left[\begin{array}{r:rr:rr:rr}
0 & 1-s & 2 & -1 & -1 & -1 & 0 \\
0 & 0 & -s & 0 & -1 & 0 & -s \\
\hdashline 0 & 0 & 0 & 0 & 0 & 1 & 0 \\
0 & 0 & 0 & 0 & 0 & 0 & 1 \\
=0 & 0 & 0 & 1 & 0 & 0 & 0 \\
0 & 0 & 0 & 0 & 1 & 0 & 0 \\
\hdashline 0 & 0 & -1 & 0 & 0 & 0 & 0
\end{array}\right],
\end{aligned}
$$

where we have included in the matrices $A_{\ell}, E_{\ell}=I, B_{\ell}, C_{\ell 1}$ and $C_{\ell 2}$ also columns corresponding to the $\mathbb{C}^{+}$zero at $s=1$ (see (59)). In this way, we solve a single Riccati equation of order 2 instead of solving a Riccati and a Lyapunov equations both of order 1 .

2. We solve the Riccati equation (21) for $X_{s}$ and compute the stabilizing feedback $F_{s}$. We get

$$
X_{s}=\frac{1}{13}\left[\begin{array}{rr}
8 & 4 \\
4 & 15
\end{array}\right], \quad F_{s}=\frac{1}{13}\left[\begin{array}{rr}
8 & 4 \\
12 & 19
\end{array}\right] .
$$

The inner factor $G_{i}(s)$ and the spectral factor $G_{o}(s)=$ $G_{i}^{-1}(s) G(s)$ are computed as follows

$$
\begin{gathered}
G_{i}(s)=Q\left[\begin{array}{c|cc}
A_{\ell}+B_{\ell} F_{s}-s E_{\ell} & B_{\ell} D_{\ell}^{-1} & -X_{s}^{-1} E_{\ell} C_{\ell 1}^{T} \\
\hline C_{\ell 1}+D_{\ell} F_{s} & I & O \\
C_{\ell 2} & O & I
\end{array}\right] \\
=\left[\begin{array}{ccc}
\frac{\left(-s-\frac{5}{13}\right)}{(s+1)} & \frac{12}{13} & 0 \\
-\frac{\frac{12}{13}}{(s+1)} & \frac{(s-1)}{(s+1)^{2}} & \frac{s}{s+1} \\
-\frac{12}{13} s \\
(s+1)^{2} & \frac{s\left(s-\frac{5}{13}\right)}{(s+1)^{2}} & \frac{1}{(s+1)}
\end{array}\right] \\
G_{o}(s)=\left[\begin{array}{ccc}
A-s E & B \\
\hline D_{\ell} H_{1} & D_{\ell} H_{2} \\
O & O
\end{array}\right] \\
=\left[\begin{array}{ccc}
-s^{2}-\frac{9}{13} s-\frac{5}{13}-4 s^{2}-\frac{23}{13} s+2 / 13-2 s^{2}+\frac{8}{13} s+\frac{34}{13} \\
s^{2}+\frac{19}{13} s+\frac{12}{13} & 4 s^{2}+\frac{63}{13} s+\frac{29}{13} & 2 s^{2}+\frac{12}{13} s-\frac{14}{13} \\
0 & 0
\end{array}\right]
\end{gathered}
$$

where

$$
\begin{gathered}
{\left[\begin{array}{rrr}
O & -F_{s} & I
\end{array}\right] Z^{-1}:=\left[\begin{array}{rr:r}
H_{1} & H_{2}
\end{array}\right]} \\
=\frac{1}{13}\left[\begin{array}{rrrrrrr}
-8 & 0 & 9 & 13 & -13 & -30 & 18 \\
-12 & 0 & -19 & -13 & 0 & -19 & -38
\end{array}\right] .
\end{gathered}
$$

\section{Conclusions}

We have given complete solutions to the inner-outer and spectral factorization problems formulated for a continuous-time system in the most general setting possible. We have provided both theoretical solutions and numerically reliable procedures.

Our approach can be viewed as a divide et impera procedure as we isolate from the original system only that subsystem which is really needed and for which we can actually solve the factorization problems. This feature of our method leads to the avoidance of unnecessary redundancy and recommends our procedure also in "standard" cases in which $G(s)$ has no zeros on the imaginary axis and is injective. Another important feature is that the subsystem in terms of which we write the Lyapunov and Riccati equations is obtained from a realization of the original system by using exclusively orthogonal transformations.

We venture to claim that there is no solution to the problem raised by Van Dooren in [40] of dislocating the minimal indices one-by-one by using all-pass factors and without a priori computing the solution to the Riccati equation (or to the Hamiltonian eigenvalue problem) of dimension $n_{\ell}$. If this were solvable, it would in general imply that a standard Riccati equation of order $n_{\ell}$ decouples into $n_{\ell}$ Riccati equations of order 1 . Yet, this negative answer should come as no surprise since even the zeros could not be dislocated in a recursive way without solving first an eigenvalue problem.

With certain changes, we can apply the methods of this paper to obtain solutions to the inner-outer and spectral factorization problems formulated for a discrete-time system [31]. Again, the same level of generality may be achieved. With minor changes we may compute also spectral factorizations with "antistable" spectral factors.

A problem actually not solved in this paper is the spectral factorization of a spectral density function which is not given in a pre-factorized form (3). As should be already clear, even if the solution to this problem will become available, and this would imply the derivation of a symmetric version of the staircase algorithm, it will be still desirable to apply the methods developed in this paper whenever $\Phi(s)$ is an already prefactorized form, or for computing the inner-outer factorization as instead of working with a $2 n$ dimensional Hermitic (or Hamiltonian) realization we manipulate only an $n$ dimensional realization of $G(s)$ featuring no symmetry.

\section{REFERENCES}

[1] B.D.O. Anderson. An algebraic solution to the spectral factorization problem. IEEE Trans. Autom. Control, AC-12:410-414, 1967.

[2] E. Anderson, Z. Bai, J. Bishop, J. Demmel, J. Du Croz, A. Greenbaum, S. Hammarling, A. McKenney, S. Ostrouchov, and D. Sorensen. LAPACK User's Guide, Second Edition. SIAM, Philadelphia, 1995.

[3] W. F. Arnold III and A. J. Laub. Generalized eigenproblem algorithms and software for algebraic Riccati equations. Proc. of IEEE, 72:1746-1754, 1984.

[4] H. Bart, I. Gohberg, M. A. Kaashoek. Constructive Methods of Wiener-Hopf Factorization. OT 21, Birkhauser, Basel, 1986.

[5] Th. Beelen and P. Van Dooren. An improved algorithm for the computation of Kronecker's canonical form of a singular pencil. Linear Algebra and its Applications, 105:9-65, 1988.

[6] P. Benner. Contributions to the numerical solution of algebraic Riccati equations and related eigenvalue problems. PhD. Dissertation. Universität Chemnitz-Zwickau, 1997.

[7] P. Benner, V. Mehrmann, V. Sima, S. Van Huffel, and A. Varga. SLICOT - a subroutine library in systems and control theory. In B. N. Datta, editor, Applied and Computational Control, Signals and Circuits, volume 1. Birkhäuser, 1997. 
[8] P. M. M. Bongers and O. H. Bosgra. Normalized coprime factorizations for systems in generalized state-space form. IEEE Trans. Autom. Control, 38:348-350, 1993.

[9] T. Chan. Rank revealing QR factorizations. Lin. Alg. \& Appl., 88/89:67-82, 1987.

[10] T. Chen and B. A. Francis. Spectral and inner-outer factorizations of rational matrices. SIAM J. Matrix Anal. Appl., 10:1-17, 1989.

[11] D. J. Clements, K. Glover. Spectral factorization by Hermitic pencils. Lin. Alg. \& Appl., 122-124:797-846, 1989.

[12] D. J. Clements, B. Anderson, A. Laub and J. Matson. Spectral factorization with imaginary axis zeros. Lin. Alg. \& Appl., 250:225-252, 1997.

[13] D. J. Clements. Rational spectral factorization using state-space methods. Syst. Contr. Lett., 335-343, 1993.

[14] J. C. Doyle. Advances in Multivariable Control. Lecture Notes, ONR/Honeywell Workshop, 1984.

[15] G. D. Forney. Minimal bases of rational vector spaces, with applications to multivariable linear systems. SIAM J. of Control, 13:493-520, 1975.

[16] F. R. Gantmacher. The Theory of Matrices. Chelsea, New York, 1960.

[17] J. D. Gardiner, A. J. Laub, J. J. Amato, and C. B. Moler. Solution of the Sylvester matrix equation $A X B^{T}+C X D^{T}=E$. ACM Trans. Math. Software, 18:223-231, 1992.

[18] M. Green. On inner-outer factorization. Syst. Contr. Letters, 11:93-97, 1988.

[19] V. Ionescu and C. Oară. Generalized continuous-time Riccati theory. Linear Algebra and Its Application, 232:111-131, 1996.

[20] V. Ionescu, C. Oară, and M. Weiss. General matrix pencil techniques for the solution of algebraic Riccati equations: A unified approach. IEEE Trans. Auto. Contr., 42:1085-1097, 1997.

[21] V. Ionescu, C. Oară, and M. Weiss. Generalized Riccati Theory and Robust Control: A Popov Function Approach. John Wiley and Sons, New York, 1999 (ISBN 047197147 2).

[22] T. Katayama. Linear quadratic regulator and spectral factorization for continuous-time descriptor systems. Proc. 31st IEEE Conf. Decision \& Contr. , Tucson, AZ, 967-972, 1992.

[23] H. Kimura. Conjugation, interpolation and model-matching in $H^{\infty}$. Int. J. Control, 49:269-307, 1989.

[24] P. Lancaster, L. Rodman. Existence and uniqueness theorems for the algebraic Riccati equation. Int. J. Control, 32:285-309, 1980.

[25] A. Laub. A Schur method for solving algebraic Riccati equations. IEEE Trans. Auto. Control, 24:913-921, 1979.

[26] V.L. Mehrmann. The Autonomous Linear Quadratic Control Problem. Vol. 163, Lecture Notes in Control and Information Sciences, Springer Verlag, New York, 1991.

[27] C. B. Moler and G. W. Stewart. An algorithm for generalized matrix eigenvalue problem. SIAM J. Numer. Anal., 10:241-256, 1973.

[28] M. Z. Nashed. Generalized Inverses and Applications. Academic Press, New York, 1976.

[29] C. Oară, P. Van Dooren. An improved algorithm for the computation of structural invariants of a system pencil and related geometric aspects. System and Control Letters, vol.30, pp. 3948, 1997.

[30] C. Oară, A. Varga. Minimal degree coprime factorization of rational matrices. SIAM Journal on Matrix Analysis and Applications, Vol.4, 1999.

[31] C. Oară, A. Varga. The general inner-outer factorization problem for discrete-time systems Proc. European Control Conference ECC'99, Karlsruhe, Germany, 1999.

[32] T. Penzl. Numerical solution of generalized Lyapunov equations. Preprint SFB393/96-02, Technical University Chemnitz, May 1996.

[33] V. M. Popov. Hyperstability of Systems. Springer, New York, 1973.

[34] H. H. Rosenbrock. State-Space and Multivariable Theory. Wiley, New York, 1970.

[35] M. G. Safonov, E. A. Jonckheere, M. Verma, and D. J. N. Limebeer. Synthesis of positive real multivariable feedback systems. Int. J. Control, 45:817-842, 1987.

[36] G. W. Stewart. Determining rank in the presence of error. in Linear Algebra for Large Scale and Real-Time Applications, M. Moonen, G. Golub and B. De Moor, Eds., Kluwer Academic Publishers, Dordrecth, 1992.
[37] P. Van Dooren. The computation of Kronecker's canonical form of a singular pencil. Lin. Alg. \& Appl., 27:103-141, 1979.

[38] P. Van Dooren. A generalized eigenvalue approach for solving Riccati equations. SIAM J. on Sci. St. Comp., 2(2):121-135, 1981.

[39] P. Van Dooren. Rational and polynomial matrix factorizations via recursive pole-zero cancellation. Internal Report Philips Research Laboratories, 1986.

[40] P. Van Dooren. Rational and polynomial matrix factorizations via recursive pole-zero cancellation. Lin. Alg. \& Appl., 137/138:663-697, 1990.

[41] A. Varga. Computation of inner-outer factorizations of rational matrices. IEEE Trans. Autom. Control, 43 (5), 1998, pp. 684688.

[42] A. Varga. Computation of Kronecker-like forms of a system pencil: Applications, algorithms and software. Proc. CACSD'96 Symposium, Dearborn, MI, pp. 77-82, 1996.

[43] A. Varga. A descriptor systems toolbox for MATLAB. Proc. CACSD'2000 Symposium, Anchorage, Alaska, 2000.

[44] G. Verghese, P. Van Dooren, and T. Kailath. Properties of the system matrix of a generalized state-space system. Int. J. Control, 30:235-243, 1979.

[45] G. Verghese, B. Lévy, and T. Kailath. A generalized state-space for singular systems. IEEE Trans. Autom. Control, AC-26:811$831,1981$.

[46] M. Weiss. Spectral and inner-outer factorizations through the constrained Riccati equation. IEEE Trans. Autom. Control, AC39:677-681, 1994.

[47] J.C. Willems. Least squares stationary optimal control and the algebraic Riccati equation. IEEE Trans. Auto. Control, 16:621$634,1971$.

[48] D.C. Youla. On the factorization of rational matrices. IRE Trans. Inform. Theory, IT-7:172-189, 1961.

[49] Z. Zhang and J. S. Freudenberg. Loop transfer recovery for non-minimum phase plants. IEEE Trans. Autom. Control, AC35:547-553, 1990.

[50] K. Zhou, J.C. Doyle, and K. Glover. Robust and Optimal Control. Prentice Hall, New Jersey, 1996.

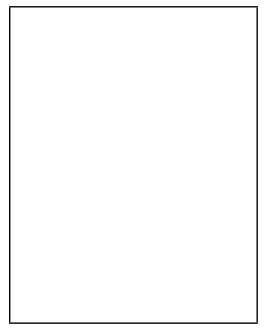

Cristian Oară was born March 1, 1968 in Bucharest, Romania. He received the University Degree and PhD Degree from the University Polytechnica Bucharest, Romania, in 1992 and 1995, respectively. From September 1995 to April 1997 he was a postdoctoral Fellow and a NATO Research Fellow at Universite Catholique de Louvain, Louvain-la-Neuve. Belgium. From May 1997 to June 1999 he was an Alexander von Humboldt Fellow at Institute for Robotics and System Dynamics, DLR

Wessling, Germany.

Since 1992 he is a staff member of the Faculty of Automatic Control and Computers, University Polytechnica Bucharest, where he is an Associate Professor of Systems Theory. His research interests include robust control and numerical linear algebra.

Dr. C. Oară is co-author of Generalized Riccati Theory and Robust Control (John Wiley \& Sons, New York, 1999). He is currently serving as an Associate Editor of the IEEE Transactions on Automatic Control. 


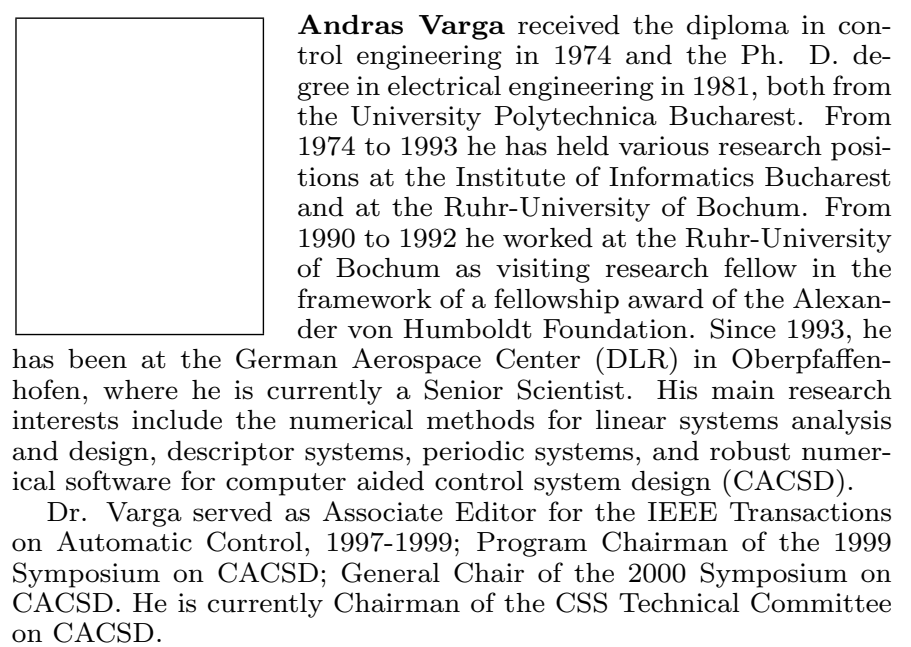

